Supporting information for

\title{
Mechanism and Dynamics of Formation of Bisoxo \\ Intermediates and O-O bond in the Catalytic Water \\ Oxidation Process
}

\section{Koteswara Rao Gorantla and Bhabani S. Mallik*}

Department of Chemistry, Indian Institute of Technology Hyderabad, Sangareddy-502285, Telangana, India

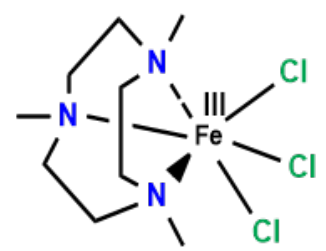

Figure S1. Catalyst 1 [ Fe( $\left.\left.\mathrm{Me}_{3} \mathrm{tacn}\right) \mathrm{Cl}_{3}\right]$, where $\mathrm{Me}_{3}$ tacn = 1,4,7-trimethyl triazacyclononane, which was reported by the Sun and co-workers. The oxygen-evolving was observed at $\mathrm{pH}=1$ in the presence of cerium ammonium nitrate as sacrificial oxidant

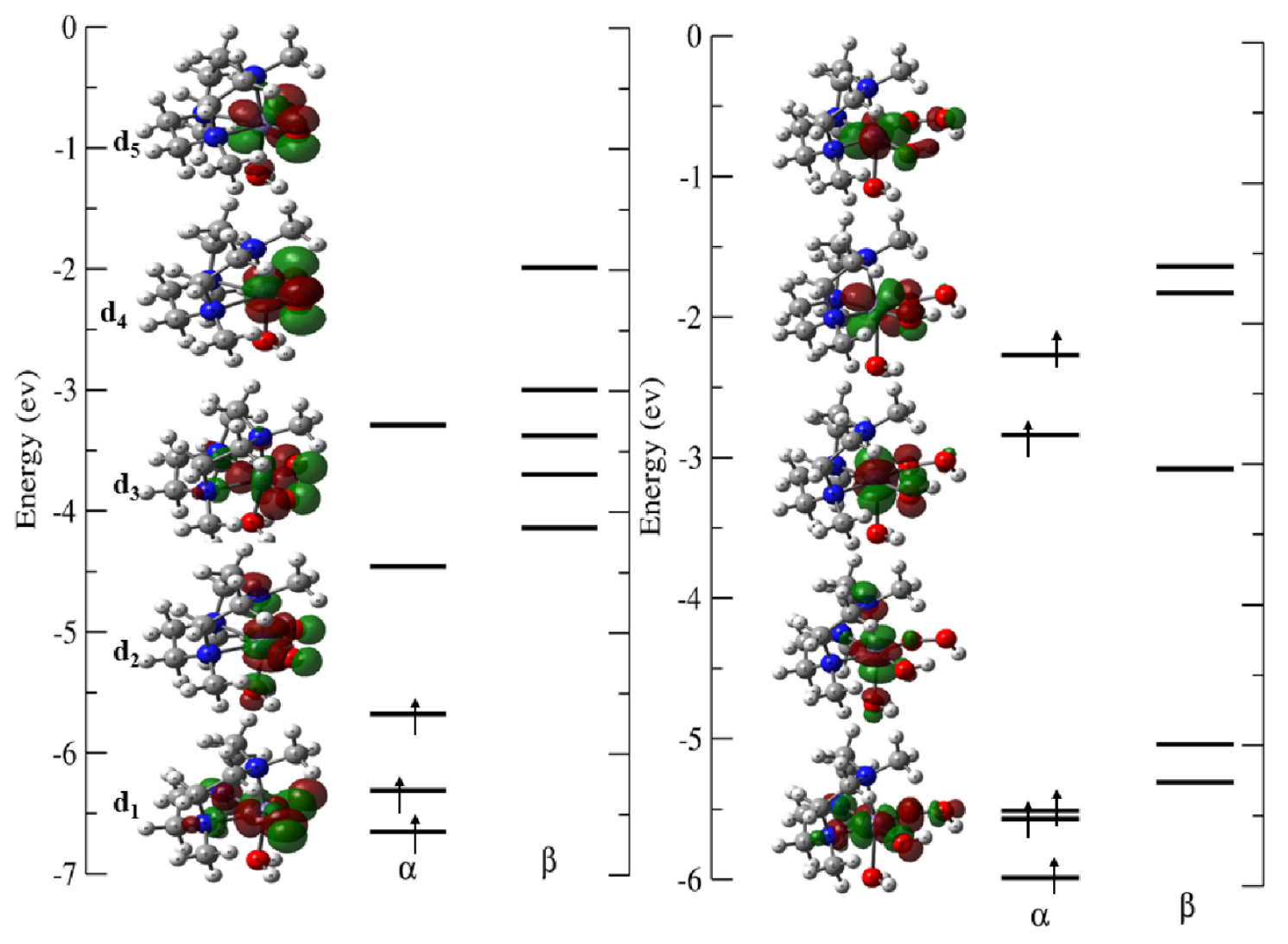

Figure S2. Molecular orbital diagram of the complex a $\left[\mathrm{FeV}\left(\mathrm{Me}_{3} \mathrm{tacn}\right)\left(\mathrm{OH}_{2}\right)(\mathrm{O})_{2}\right]^{+}$and complex b $\left[\mathrm{Fe}^{\mathrm{III}}\left(\mathrm{Me}_{3} \mathrm{tacn}\right)\left(\mathrm{OH}_{2}\right)(\mathrm{OOH})(\mathrm{OH})\right]^{+}$(before and after the formation of oxygen-oxygen bond formation 


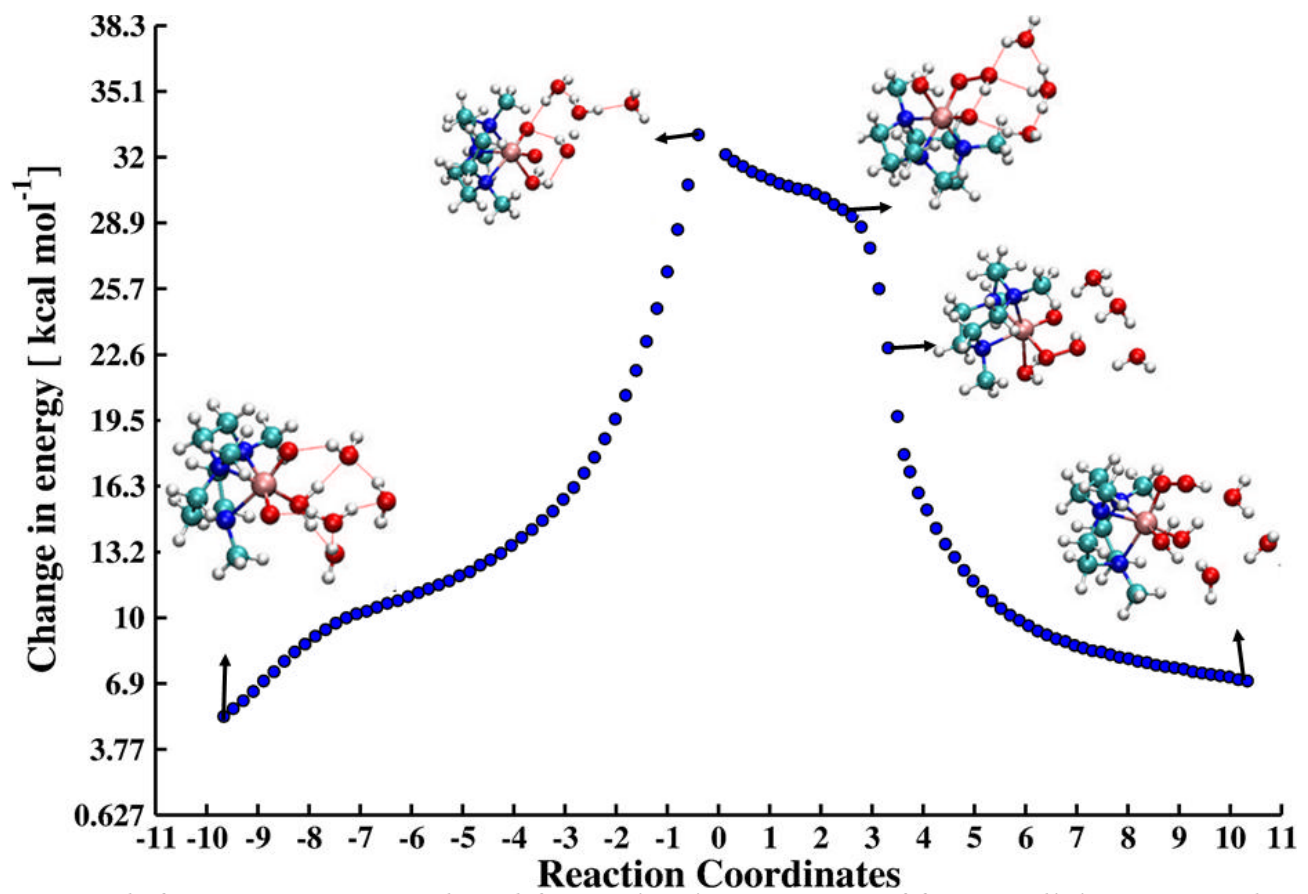

Figure S3. IRC path for oxygen-oxygen bond formation in presence of four explicit water molecules. Carried at Mo6L/SDD for Fe/6-31+ $\mathrm{G}^{*}$ for other in PCM (solvent model) $\left[\mathrm{Fe}^{\mathrm{V}}\left(\mathrm{Me}_{3} \mathrm{tacn}\right)\left(\mathrm{H}_{2} \mathrm{O}\right)(\mathrm{O})_{2}\right]$ as the starting one with four explicit water molecule.

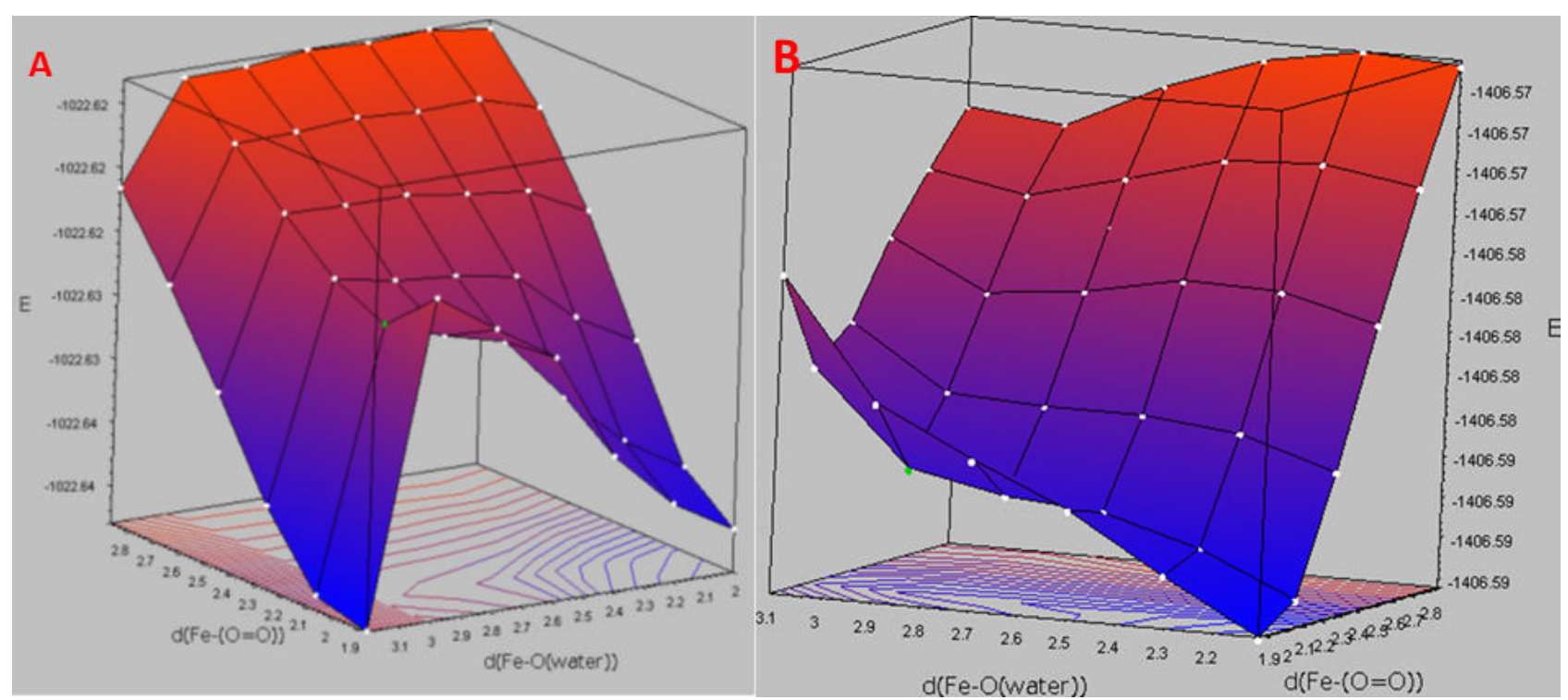

Figure S4. The potential energy scan (PES) for the release of oxygen with addition of water molecule from A) $\left[\mathrm{Fe}^{\mathrm{III}}\left(\mathrm{Me}_{3} \mathrm{tacn}\right)\left(\mathrm{H}_{2} \mathrm{O}\right)(\mathrm{OO})(\mathrm{OH})\right]^{+}$and $\left.\mathrm{B}\right)$ for $\left[\mathrm{Fe}^{\mathrm{III}}\left(\mathrm{Me}_{3} \mathrm{tacn}\right)(\mathrm{Cl})(\mathrm{OO})(\mathrm{OH})\right]$ 


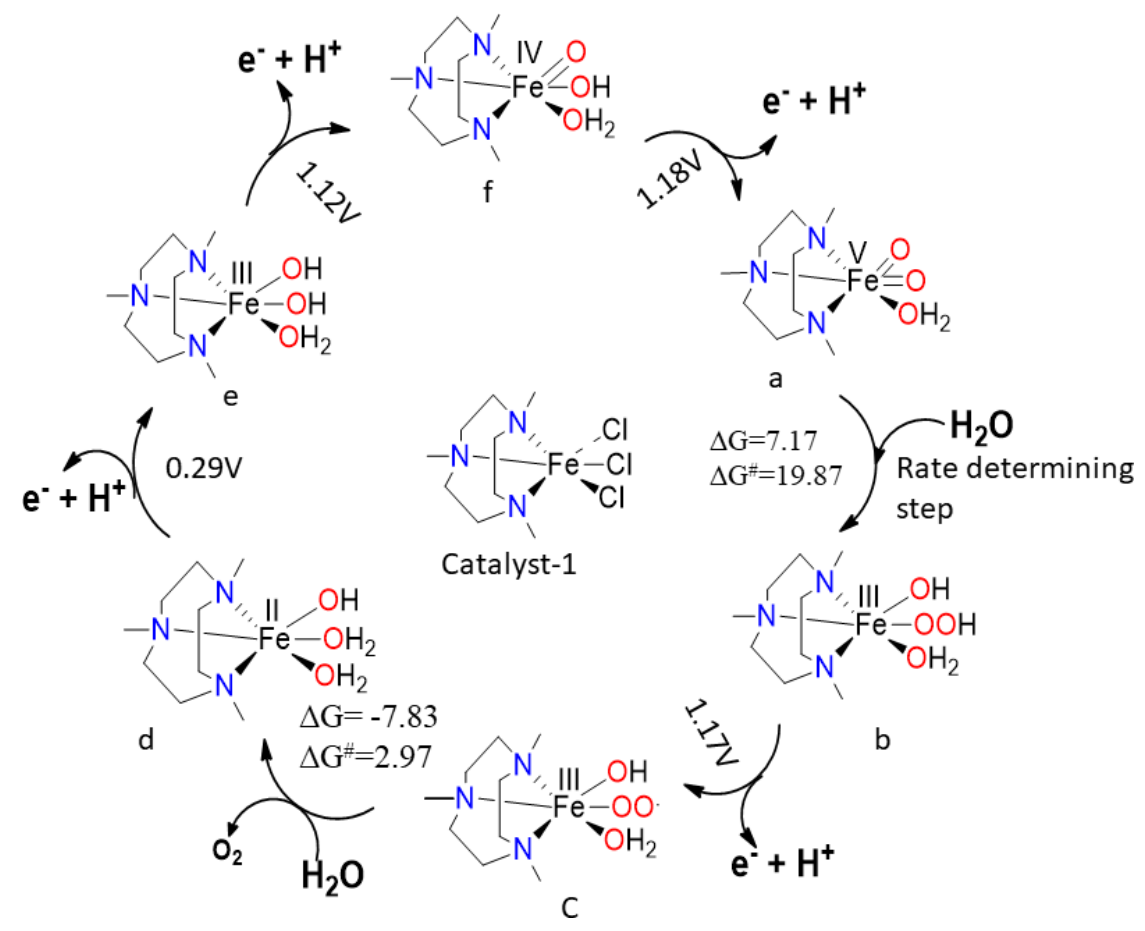

Figure S5. Total catalytic cycle (free energies and activation free energies are in kcal.mol-1) for $\left[\mathrm{Fe}^{\mathrm{V}}\left(\mathrm{Me}_{3} \mathrm{tacn}\right)\right.$ $\left.\left(\mathrm{H}_{2} \mathrm{O}\right)(\mathrm{O})_{2}\right]^{+}$as a starting one

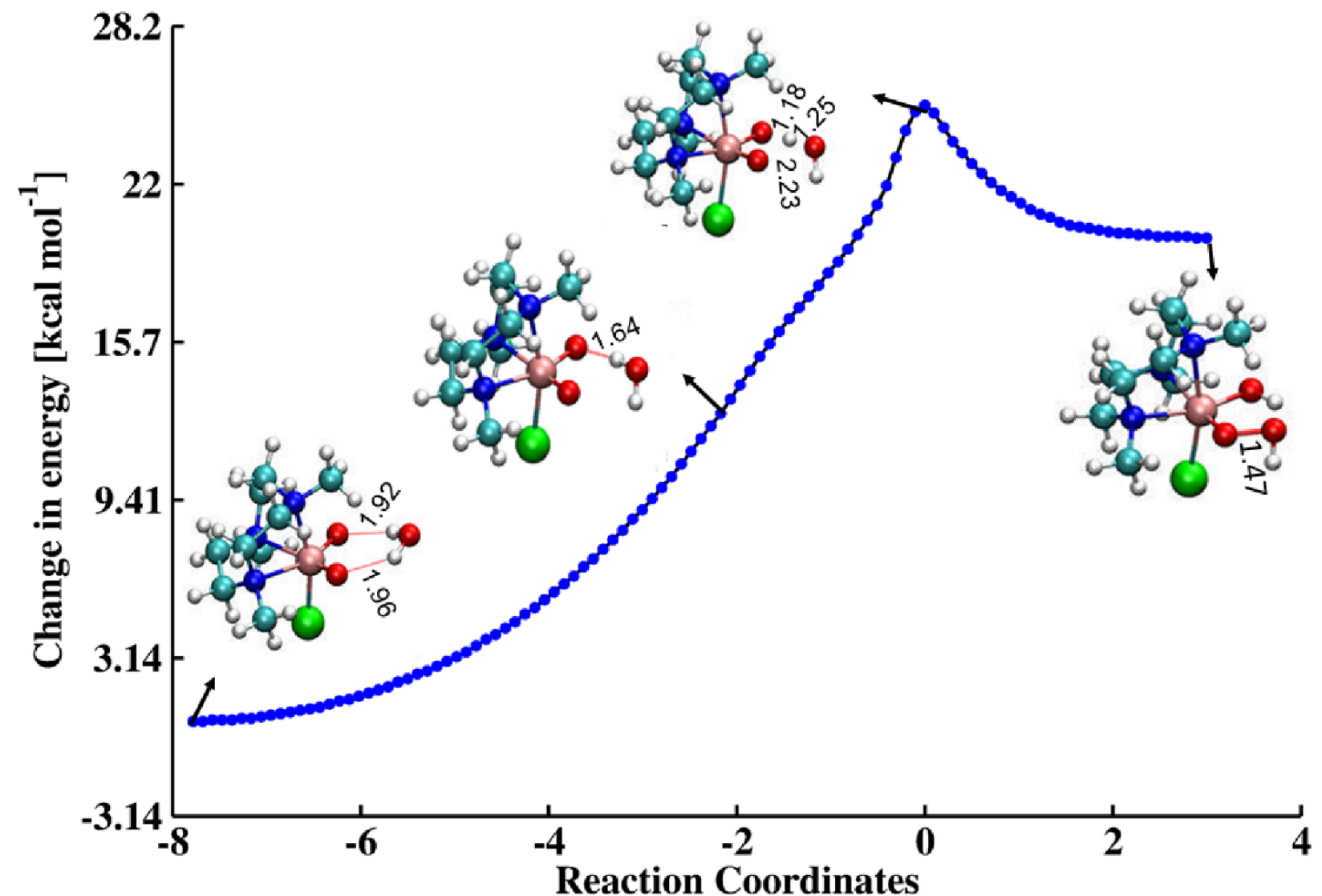

Figure S6. IRC path for oxygen-oxygen bond formation. Carried at Mo6L/SDD for Fe/6-31+G* for other in PCM (solvent model) $\left[\mathrm{FeV}^{\mathrm{V}}\left(\mathrm{Me}_{3} \mathrm{tacn}\right)(\mathrm{Cl})(\mathrm{O})_{2}\right]^{+}$as the starting one 

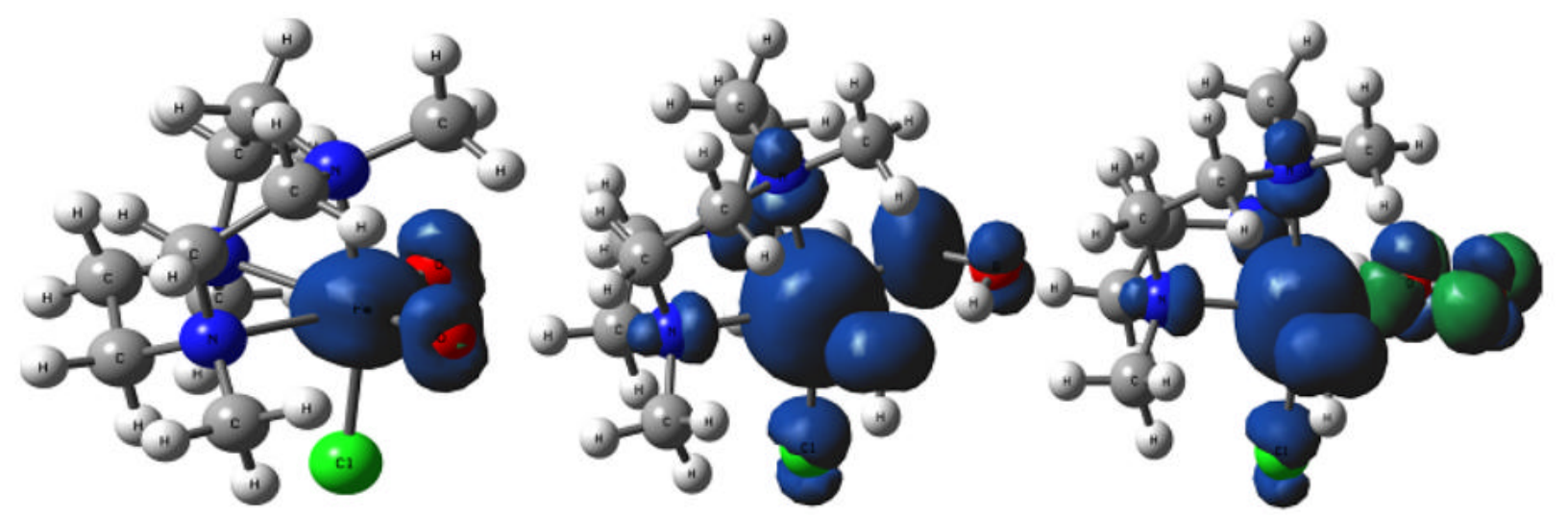

Figure S7. spin density of complex a $\left[\mathrm{Fe}^{\mathrm{V}}\left(\mathrm{Me}_{3} \mathrm{tacn}\right)(\mathrm{Cl})(\mathrm{O})_{2}\right]^{+}$, complex b $\left[\mathrm{Fe}^{\mathrm{III}}\left(\mathrm{Me}_{3} \mathrm{tacn}\right)(\mathrm{Cl})(\mathrm{OOH})(\mathrm{OH})\right]^{+}$and superoxide complex $\left[\mathrm{Fe}^{\mathrm{III}}\left(\mathrm{Me}_{3} \mathrm{tacn}\right)(\mathrm{Cl})(\mathrm{OO})(\mathrm{OH})\right]^{+}$(with isovalue -0.04)

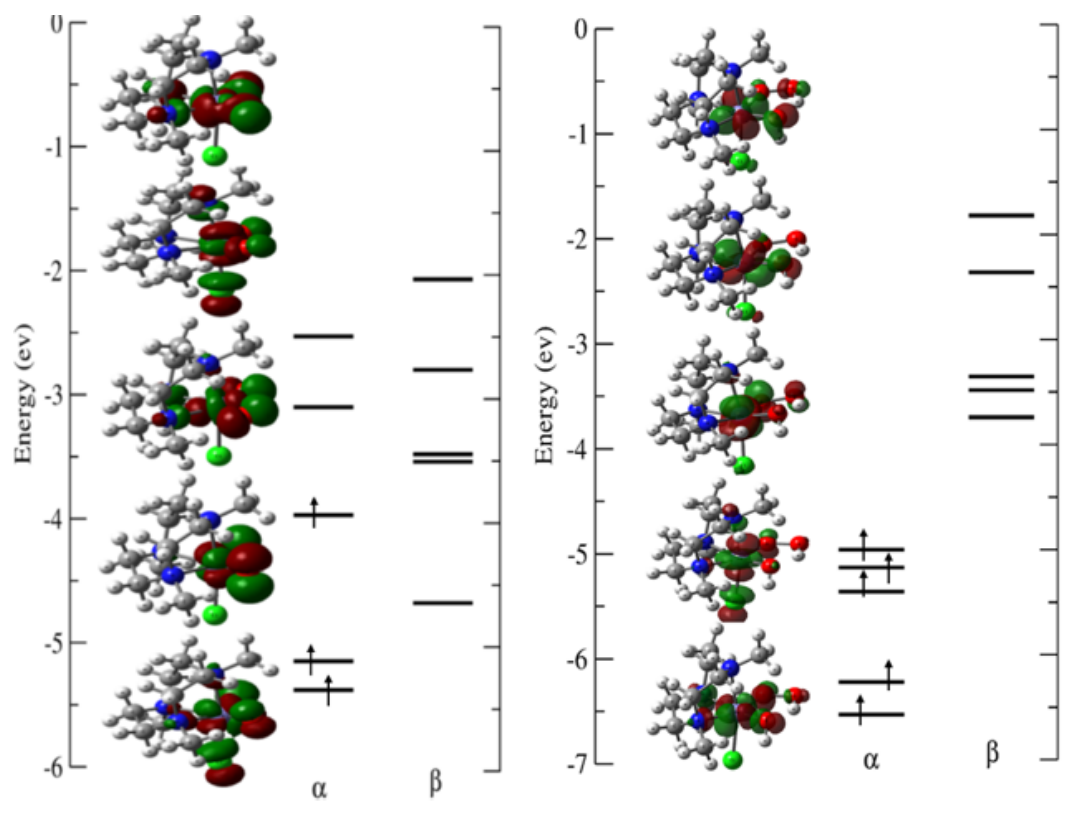

Figure S8. Molecular orbital diagram of complex a $\left[\mathrm{FeV}^{\mathrm{V}}\left(\mathrm{Me}_{3} \mathrm{tacn}\right)(\mathrm{Cl})(\mathrm{O})_{2}\right]^{+}$and complex b $\left[\mathrm{Fe}^{\mathrm{III}}\left(\mathrm{Me}_{3} \mathrm{tacn}\right)(\mathrm{Cl})(\mathrm{OOH})(\mathrm{OH})\right]^{+}$(before and after formation of oxygen-oxygen bond formation)

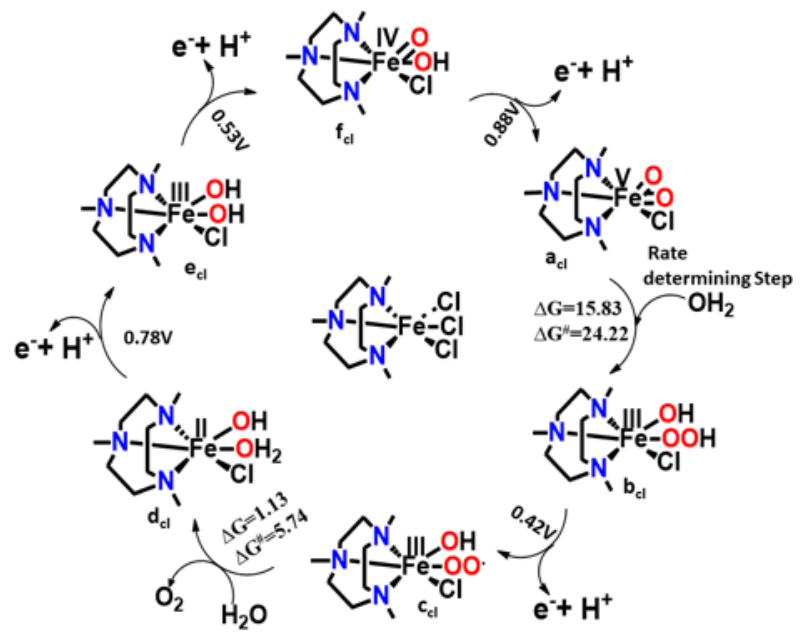

Figure S9. Total catalytic cycle (free energies and activation free energies are in kcal.mol-1) for $\left[\mathrm{Fev}^{\mathrm{V}}\left(\mathrm{Me}_{3} \mathrm{tacn}\right)(\mathrm{Cl})(\mathrm{O})_{2}\right]^{+}$as a starting one. 

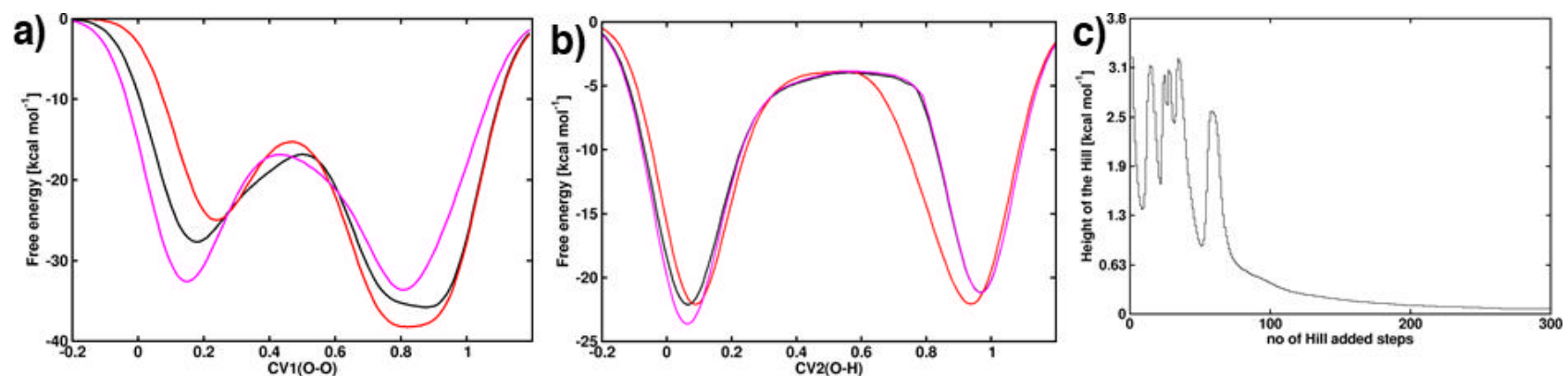

Figure S10. Reconstruction of the free energies along the single collective variables A) MFEP along the CV1 B) MFEP along the CV2 of the FES shown in the Figure 7. C) Propagation of the hills during the welltempered Metadynamics Simulations
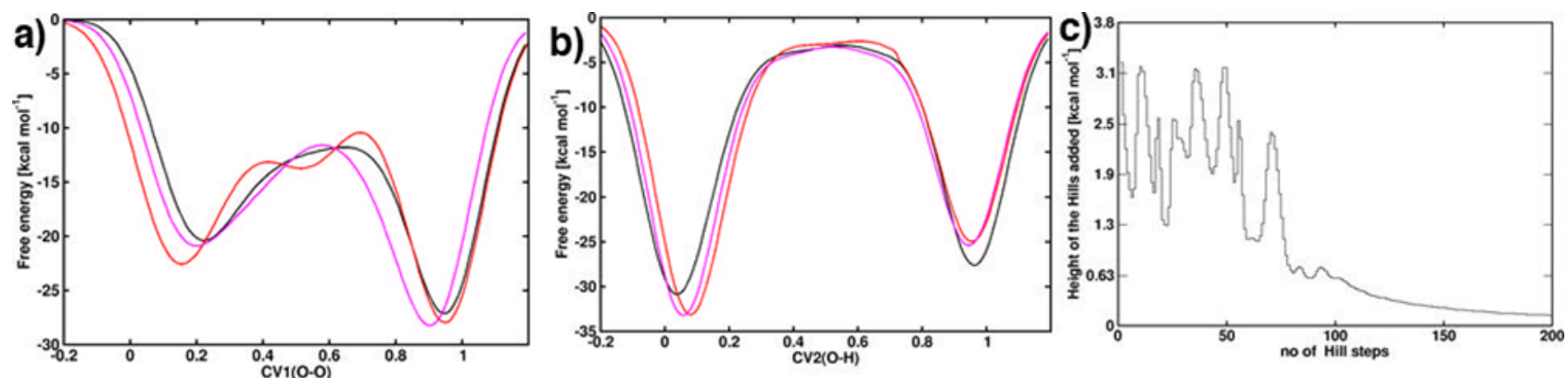

Figure S11. Reconstruction of the free energies along the single collective variables A) MFEP along the CV1 B) MFEP along the CV2 of the FES shown in the Figure 8. C) Propagation of the hills during the welltempered Metadynamics Simulations

\section{Cartesian coordinates of intermediates and transition states}

\section{$\left[\mathrm{Fe}\left(\mathrm{Me}_{3} \operatorname{tacn}\right)(\mathrm{O})_{2}\right]^{+}$}

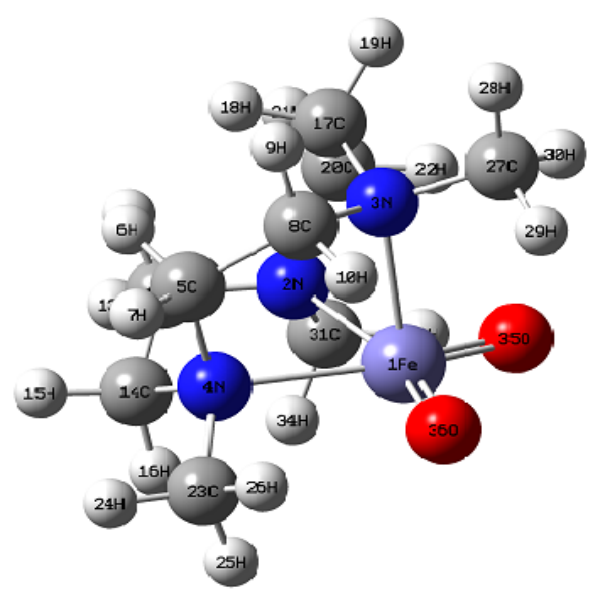

$\begin{array}{cccc}\text { atom } & \mathrm{X} & \mathrm{Y} & \mathrm{Z} \\ \mathrm{Fe} & 0.00160800 & 0.30082200 & -1.03633800 \\ \mathrm{~N} & -1.41800400 & -0.94841100 & 0.17418200 \\ \mathrm{~N} & 0.04511900 & 1.45421200 & 0.74145800 \\ \mathrm{~N} & 1.37001100 & -1.02640500 & 0.14475000 \\ \mathrm{C} & 1.71262400 & -0.27168700 & 1.38549000 \\ \mathrm{H} & 1.16320900 & -0.70317800 & 2.22616300 \\ \mathrm{H} & 2.77397700 & -0.40644100 & 1.61909900 \\ \mathrm{C} & 1.41568600 & 1.21403800 & 1.25948600 \\ \mathrm{H} & 1.54248400 & 1.70238100 & 2.23557300 \\ \mathrm{H} & 2.10121400 & 1.68619700 & 0.55091000 \\ \mathrm{C} & -0.73007800 & -1.96314600 & 1.01329300\end{array}$




$\begin{array}{lccc}\mathrm{H} & -0.64403100 & -1.58627800 & 2.03587900 \\ \mathrm{H} & -1.33271200 & -2.87517200 & 1.07861400 \\ \mathrm{C} & 0.63089600 & -2.27511300 & 0.43292100 \\ \mathrm{H} & 1.20356300 & -2.92858300 & 1.10609600 \\ \mathrm{H} & 0.52779200 & -2.80828300 & -0.51953100 \\ \mathrm{C} & -1.02443400 & 0.99043400 & 1.67308600 \\ \mathrm{H} & -0.55333200 & 0.45006500 & 2.49899200 \\ \mathrm{H} & -1.51999600 & 1.85839400 & 2.11905900 \\ \mathrm{C} & -2.05942100 & 0.12140100 & 0.97467800 \\ \mathrm{H} & -2.74822300 & -0.30479400 & 1.71730600 \\ \mathrm{H} & -2.64628400 & 0.72046900 & 0.27301500 \\ \mathrm{C} & 2.59265300 & -1.32813200 & -0.62697700 \\ \mathrm{H} & 3.24260900 & -2.00758100 & -0.06247600 \\ \mathrm{H} & 2.32383100 & -1.79245300 & -1.57742100 \\ \mathrm{H} & 3.12073000 & -0.40023600 & -0.84440100 \\ \mathrm{C} & -0.14136300 & 2.86727200 & 0.34847900 \\ \mathrm{H} & -0.02138300 & 3.52241200 & 1.21892700 \\ \mathrm{H} & 0.59428100 & 3.12576000 & -0.41461500 \\ \mathrm{H} & -1.13799100 & 2.98972000 & -0.07687000 \\ \mathrm{C} & -2.40741700 & -1.59686800 & -0.71029000 \\ \mathrm{H} & -3.20596900 & -2.06256900 & -0.12048100 \\ \mathrm{H} & -2.83127200 & -0.85461600 & -1.38606000 \\ \mathrm{H} & -1.91229200 & -2.37050400 & -1.30209600 \\ \mathrm{O} & -1.33356500 & 0.88965400 & -1.71318100 \\ \mathrm{O} & 1.33397000 & 0.90021400 & -1.70614700 \\ & & & \end{array}$

\section{$\left[\mathrm{Fe}\left(\mathrm{Me}_{3} \mathrm{tacn}\right)\left(\mathrm{OH}_{2}\right)(\mathrm{O})_{2}\right]^{+}$}

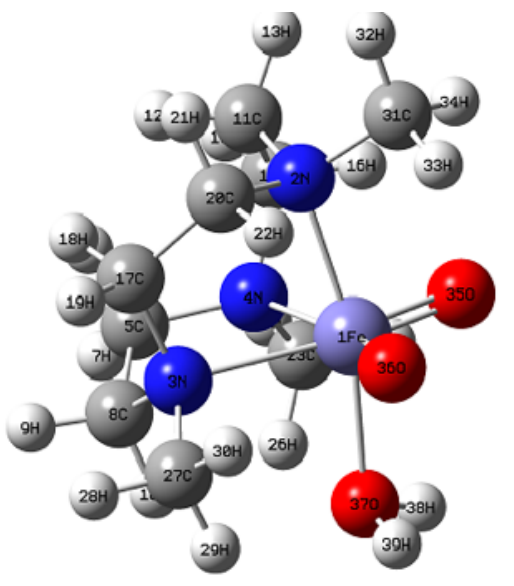

$\begin{array}{cccc}\mathrm{Fe} & -0.05600000 & 0.00500000 & -0.94200000 \\ \mathrm{~N} & -1.69400000 & -0.00100000 & 0.30100000 \\ \mathrm{~N} & 0.75100000 & 1.38500000 & 0.65100000 \\ \mathrm{~N} & 0.74800000 & -1.41000000 & 0.62100000 \\ \mathrm{C} & 1.39700000 & -0.70900000 & 1.76100000 \\ \mathrm{H} & 0.69400000 & -0.65200000 & 2.59600000 \\ \mathrm{H} & 2.24900000 & -1.29000000 & 2.12700000 \\ \mathrm{C} & 1.85000000 & 0.66800000 & 1.33400000 \\ \mathrm{H} & 2.22000000 & 1.24000000 & 2.19600000 \\ \mathrm{H} & 2.66900000 & 0.59100000 & 0.61200000 \\ \mathrm{C} & -1.57500000 & -1.07200000 & 1.34800000 \\ \mathrm{H} & -1.36400000 & -0.59000000 & 2.30500000\end{array}$




$\begin{array}{lccc}\mathrm{H} & -2.54300000 & -1.56700000 & 1.45700000 \\ \mathrm{C} & -0.50900000 & -2.08800000 & 1.00200000 \\ \mathrm{H} & -0.35400000 & -2.77000000 & 1.84800000 \\ \mathrm{H} & -0.81600000 & -2.68100000 & 0.13700000 \\ \mathrm{C} & -0.38900000 & 1.68600000 & 1.56000000 \\ \mathrm{H} & -0.27000000 & 1.11900000 & 2.48500000 \\ \mathrm{H} & -0.37500000 & 2.74000000 & 1.85100000 \\ \mathrm{C} & -1.71200000 & 1.36700000 & 0.89800000 \\ \mathrm{H} & -2.53400000 & 1.43500000 & 1.62000000 \\ \mathrm{H} & -1.91300000 & 2.05600000 & 0.07400000 \\ \mathrm{C} & 1.67800000 & -2.37100000 & 0.01000000 \\ \mathrm{H} & 1.89400000 & -3.19900000 & 0.69500000 \\ \mathrm{H} & 1.23000000 & -2.76400000 & -0.90500000 \\ \mathrm{H} & 2.61600000 & -1.86500000 & -0.22900000 \\ \mathrm{C} & 1.25300000 & 2.62500000 & 0.04100000 \\ \mathrm{H} & 1.62600000 & 3.31300000 & 0.81000000 \\ \mathrm{H} & 2.07000000 & 2.38900000 & -0.64300000 \\ \mathrm{H} & 0.44800000 & 3.09700000 & -0.52300000 \\ \mathrm{C} & -2.89700000 & -0.21100000 & -0.53100000 \\ \mathrm{H} & -3.79500000 & -0.09300000 & 0.08500000 \\ \mathrm{H} & -2.89800000 & 0.52200000 & -1.33900000 \\ \mathrm{H} & -2.86300000 & -1.21400000 & -0.95600000 \\ \mathrm{O} & -0.49100000 & -1.33300000 & -1.78200000 \\ \mathrm{O} & -0.57200000 & 1.32300000 & -1.76600000 \\ \mathrm{O} & 1.86600000 & 0.10500000 & -1.77900000 \\ \mathrm{H} & 2.01000000 & -0.70100000 & -2.29700000 \\ \mathrm{H} & 1.81300000 & 0.81200000 & -2.44000000\end{array}$

$\left[\mathrm{Fe}\left(\mathrm{Me}_{3} \mathrm{tacn}\right)\left(\mathrm{OH}_{2}\right)(\mathrm{O})_{2}\right]^{+} \mathrm{H}_{2} \mathrm{O}$

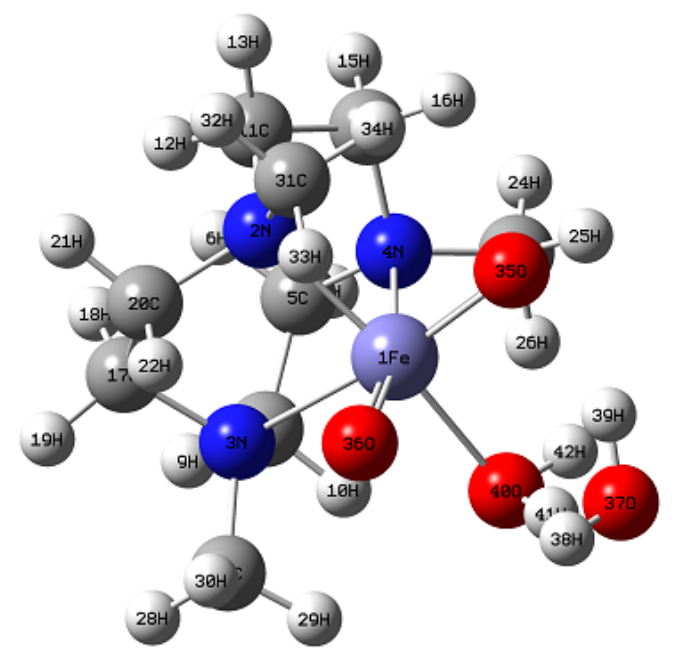

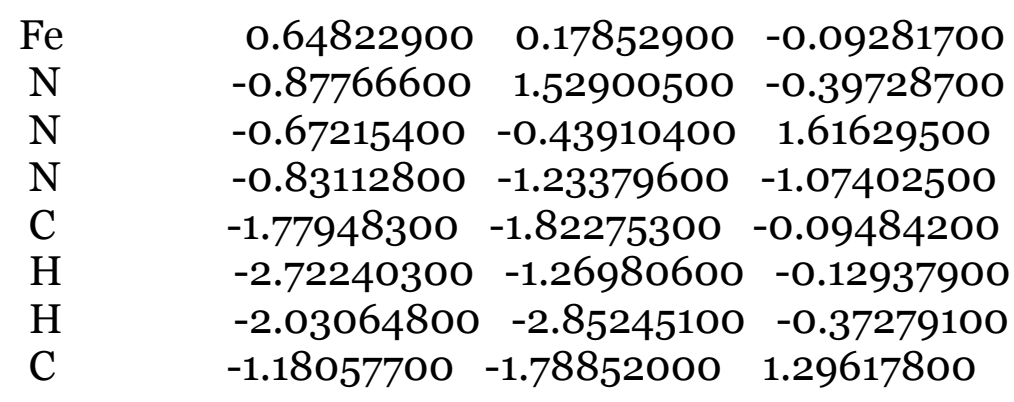




\begin{tabular}{|c|c|c|c|}
\hline $\mathrm{H}$ & -1.91391700 & -2.12839000 & 2.04226400 \\
\hline $\mathrm{H}$ & -0.32123000 & -2.46500500 & 1.35589200 \\
\hline $\mathrm{C}$ & -1.94540800 & 0.94974200 & -1.27696000 \\
\hline $\mathrm{H}$ & -2.81541800 & 0.72331100 & -0.65551900 \\
\hline $\mathrm{H}$ & -2.26798900 & 1.71024700 & -1.99417100 \\
\hline $\mathrm{C}$ & -1.46676500 & -0.28423900 & -2.01162600 \\
\hline $\mathrm{H}$ & -2.30542400 & -0.75109800 & -2.54821800 \\
\hline $\mathrm{H}$ & -0.70015600 & -0.01313200 & -2.74282000 \\
\hline 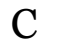 & -1.75497800 & 0.57477700 & 1.71617400 \\
\hline $\mathrm{H}$ & -2.68448100 & 0.14990800 & 1.32631000 \\
\hline $\mathrm{H}$ & -1.95509500 & 0.81632900 & 2.76596600 \\
\hline $\mathrm{C}$ & -1.38361300 & 1.84098000 & 0.97087800 \\
\hline $\mathrm{H}$ & -2.24596600 & 2.51682300 & 0.89574900 \\
\hline $\mathrm{H}$ & -0.57432900 & 2.36831600 & 1.48095000 \\
\hline $\mathrm{C}$ & -0.13046000 & -2.28210700 & -1.82895500 \\
\hline $\mathrm{H}$ & -0.80507800 & -2.79368300 & -2.52706800 \\
\hline $\mathrm{H}$ & 0.68874100 & -1.82338000 & -2.38996100 \\
\hline $\mathrm{H}$ & 0.25955800 & -3.03480900 & -1.13703700 \\
\hline $\mathrm{C}$ & 0.12443700 & -0.47008500 & 2.85511900 \\
\hline $\mathrm{H}$ & -0.48988100 & -0.79308000 & 3.70523700 \\
\hline $\mathrm{H}$ & 0.96005900 & -1.15938000 & 2.72683400 \\
\hline $\mathrm{H}$ & 0.52886000 & 0.52500500 & 3.04189800 \\
\hline $\mathrm{C}$ & -0.28608700 & 2.73794900 & -1.01406800 \\
\hline $\mathrm{H}$ & -1.04308000 & 3.52692200 & -1.08534700 \\
\hline $\mathrm{H}$ & 0.55119100 & 3.06986500 & -0.39927300 \\
\hline $\mathrm{H}$ & 0.08742400 & 2.47990100 & -2.00515000 \\
\hline $\mathrm{O}$ & 1.32236300 & 0.36333600 & -1.57934400 \\
\hline $\mathrm{O}$ & 1.42694600 & 1.20253300 & 0.91852100 \\
\hline $\mathrm{O}$ & $3 \cdot 91252200$ & 0.12681600 & -0.15015500 \\
\hline $\mathrm{H}$ & 3.98145100 & 0.81799800 & 0.51878000 \\
\hline $\mathrm{H}$ & 3.40251700 & 0.54957100 & -0.86027100 \\
\hline $\mathrm{O}$ & 1.88152900 & -1.39856300 & 0.40076100 \\
\hline $\mathrm{H}$ & 2.75961800 & -0.89113000 & 0.34968200 \\
\hline $\mathrm{H}$ & 1.93906800 & -2.00078200 & -0.35386000 \\
\hline
\end{tabular}

\section{$\left[\mathrm{Fe}\left(\mathrm{Me}_{3} \operatorname{tacn}\right)\left(\mathrm{OH}_{2}\right)(\mathrm{OH})(\mathrm{OOH})\right]^{+}$}

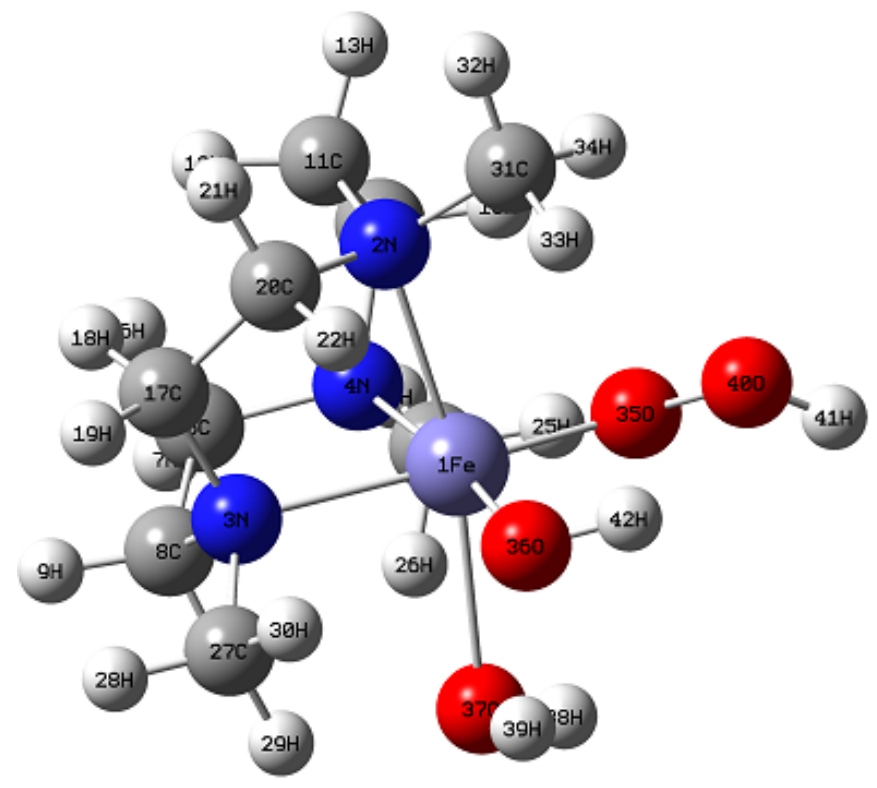




\begin{tabular}{|c|c|c|c|}
\hline $\mathrm{Fe}$ & 0.32400000 & 0.52300000 & -0.28200000 \\
\hline $\mathrm{N}$ & 0.64600000 & -1.68700000 & -0.23100000 \\
\hline $\mathrm{N}$ & -1.66100000 & -0.08600000 & -0.75000000 \\
\hline $\mathrm{N}$ & -0.32100000 & 0.14000000 & 1.70700000 \\
\hline $\mathrm{C}$ & -1.78700000 & -0.15600000 & 1.71300000 \\
\hline $\mathrm{H}$ & -1.92900000 & -1.23300000 & 1.83000000 \\
\hline $\mathrm{H}$ & -2.26000000 & 0.30600000 & 2.58600000 \\
\hline $\mathrm{C}$ & -2.43700000 & 0.34600000 & 0.44100000 \\
\hline $\mathrm{H}$ & -3.48000000 & 0.00300000 & 0.37800000 \\
\hline $\mathrm{H}$ & -2.44600000 & 1.44000000 & 0.42400000 \\
\hline $\mathrm{C}$ & 0.38800000 & -2.17000000 & 1.14300000 \\
\hline $\mathrm{H}$ & -0.60200000 & -2.63400000 & 1.18600000 \\
\hline $\mathrm{H}$ & 1.09900000 & -2.95900000 & 1.41600000 \\
\hline $\mathrm{C}$ & 0.49700000 & -1.02600000 & 2.13700000 \\
\hline $\mathrm{H}$ & 0.20400000 & -1.35400000 & 3.14400000 \\
\hline $\mathrm{H}$ & 1.53100000 & -0.66700000 & 2.18400000 \\
\hline $\mathrm{C}$ & -1.70800000 & -1.56900000 & -0.94700000 \\
\hline $\mathrm{H}$ & -2.14500000 & -2.02900000 & -0.05600000 \\
\hline $\mathrm{H}$ & -2.38700000 & -1.80900000 & -1.77100000 \\
\hline $\mathrm{C}$ & -0.33400000 & -2.14100000 & -1.23300000 \\
\hline $\mathrm{H}$ & -0.38800000 & -3.23900000 & -1.28700000 \\
\hline $\mathrm{H}$ & 0.02500000 & -1.77700000 & -2.20200000 \\
\hline $\mathrm{C}$ & -0.02400000 & 1.30500000 & 2.56200000 \\
\hline $\mathrm{H}$ & -0.23000000 & 1.08100000 & 3.61600000 \\
\hline $\mathrm{H}$ & 1.02800000 & 1.57300000 & 2.44900000 \\
\hline $\mathrm{H}$ & -0.65000000 & 2.14600000 & 2.25400000 \\
\hline $\mathrm{C}$ & -2.16500000 & 0.59400000 & -1.96100000 \\
\hline $\mathrm{H}$ & -3.19700000 & 0.28900000 & -2.17100000 \\
\hline $\mathrm{H}$ & -2.14300000 & 1.67400000 & -1.80900000 \\
\hline $\mathrm{H}$ & -1.51800000 & 0.35000000 & -2.80500000 \\
\hline $\mathrm{C}$ & 2.02100000 & -1.98400000 & -0.65800000 \\
\hline $\mathrm{H}$ & 2.18300000 & -3.06600000 & -0.75200000 \\
\hline $\mathrm{H}$ & 2.21100000 & -1.50400000 & -1.62000000 \\
\hline $\mathrm{H}$ & 2.72600000 & -1.56900000 & 0.06500000 \\
\hline $\mathrm{O}$ & 1.93600000 & 1.05400000 & 0.35700000 \\
\hline $\mathrm{O}$ & 0.68900000 & 0.89000000 & -2.02200000 \\
\hline $\mathrm{O}$ & -0.49300000 & 2.75400000 & -0.31000000 \\
\hline $\mathrm{H}$ & 0.10800000 & 3.33900000 & 0.16600000 \\
\hline $\mathrm{H}$ & -0.16400000 & 2.76400000 & -1.22500000 \\
\hline $\mathrm{O}$ & 3.04300000 & 0.90200000 & -0.58800000 \\
\hline $\mathrm{H}$ & 3.58900000 & 1.66000000 & -0.33400000 \\
\hline $\mathrm{H}$ & 1.64400000 & 1.06100000 & -2.08000000 \\
\hline
\end{tabular}

$\left[\mathrm{Fe}\left(\mathrm{Me}_{3} \text { tacn }\right)\left(\mathrm{OH}_{2}\right)(\mathrm{OO})(\mathrm{OH})\right]^{+}$ 


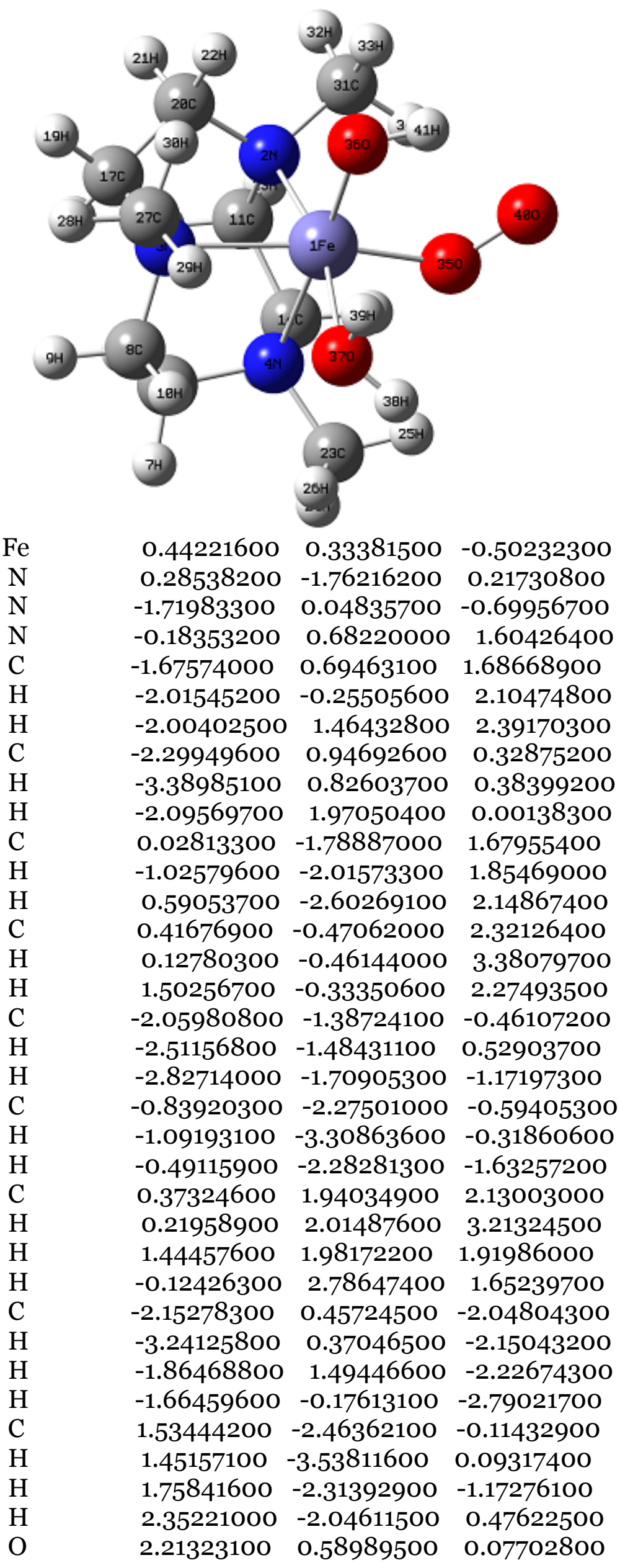




\begin{tabular}{|c|c|c|c|}
\hline $\mathrm{O}$ & 0.89126500 & -0.02356500 & -2.24531900 \\
\hline $\mathrm{O}$ & 0.11565200 & 2.49604800 & -0.96317200 \\
\hline $\mathrm{H}$ & 0.75485500 & 3.09377800 & -0.55391400 \\
\hline $\mathrm{H}$ & 0.33003400 & 2.52888300 & -1.90622600 \\
\hline $\mathrm{O}$ & 3.25972200 & 0.51239400 & -0.68033900 \\
\hline $\mathrm{H}$ & 1.85122100 & 0.01713800 & -2.37337400 \\
\hline
\end{tabular}

$\left[\mathrm{Fe}\left(\mathrm{Me}_{3} \operatorname{tacn}\right)\left(\mathrm{OH}_{2}\right)\left(\mathrm{H}_{2} \mathrm{O}\right)(\mathrm{OH})\right]^{+}$

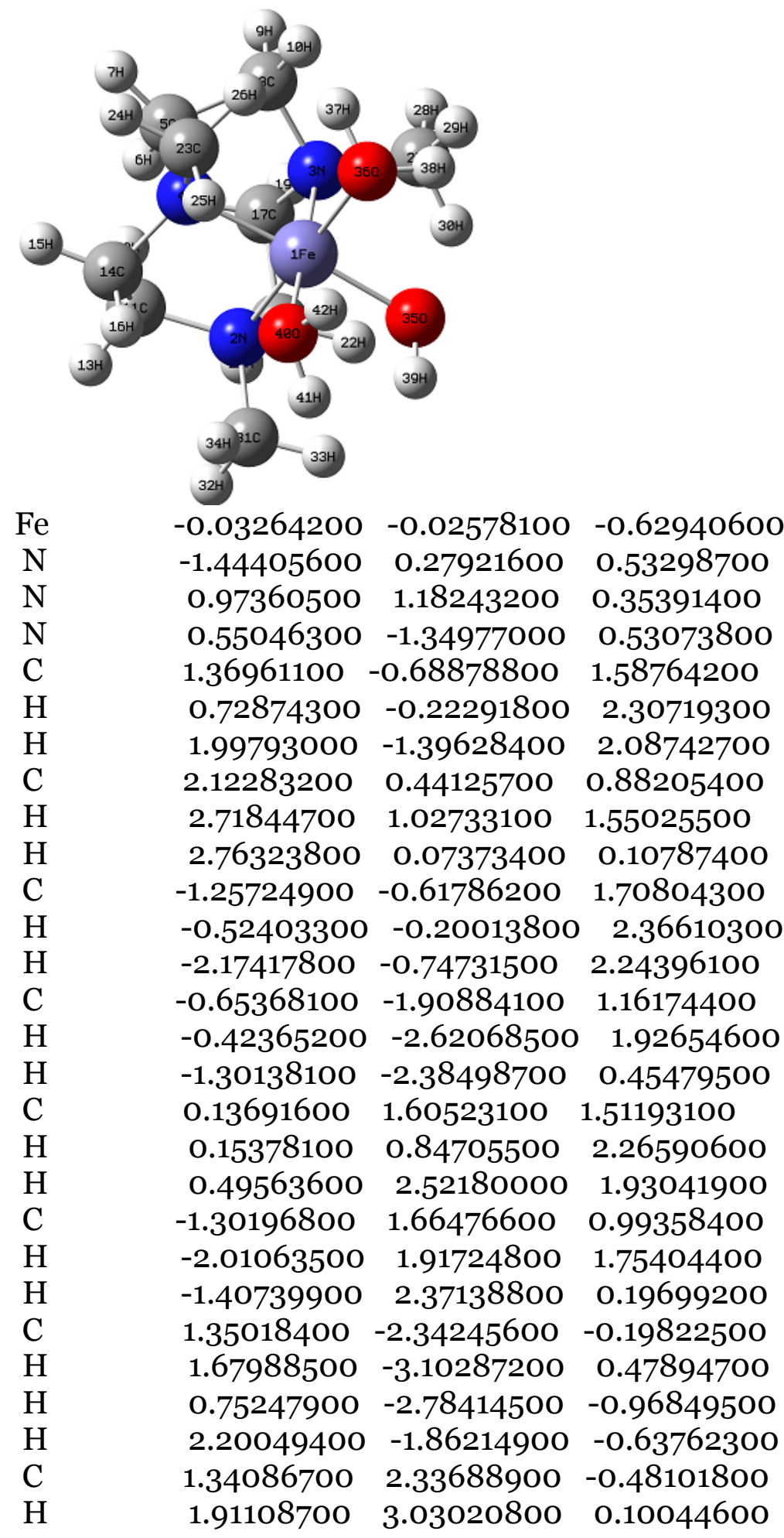




$\begin{array}{lccl}\mathrm{H} & 1.92579400 & 2.00257600 & -1.31429700 \\ \mathrm{H} & 0.45392200 & 2.81629100 & -0.83852000 \\ \mathrm{C} & -2.72460700 & 0.01400000 & -0.14087900 \\ \mathrm{H} & -3.52910700 & 0.20023500 & 0.53853500 \\ \mathrm{H} & -2.81955600 & 0.65627000 & -0.99140600 \\ \mathrm{H} & -2.75724400 & -1.00644100 & -0.46014300 \\ \mathrm{O} & -0.70198100 & 1.23669800 & -1.75555200 \\ \mathrm{O} & 1.35236200 & -0.32497200 & -1.77024700 \\ \mathrm{H} & 2.13800100 & -0.55136200 & -1.26616600 \\ \mathrm{H} & 1.51914800 & 0.46694400 & -2.28601900 \\ \mathrm{H} & -1.46389100 & 0.87902700 & -2.21692200 \\ \mathrm{O} & -0.98769500 & -1.24989100 & -1.57911300 \\ \mathrm{H} & -1.83614100 & -0.87446900 & -1.82620000 \\ \mathrm{H} & -0.50167800 & -1.48905100 & -2.37254500\end{array}$

$\left[\mathrm{Fe}\left(\mathrm{Me}_{3} \mathrm{tacn}\right)\left(\mathrm{OH}_{2}\right)(\mathrm{OH})(\mathrm{OH})\right]^{+}$

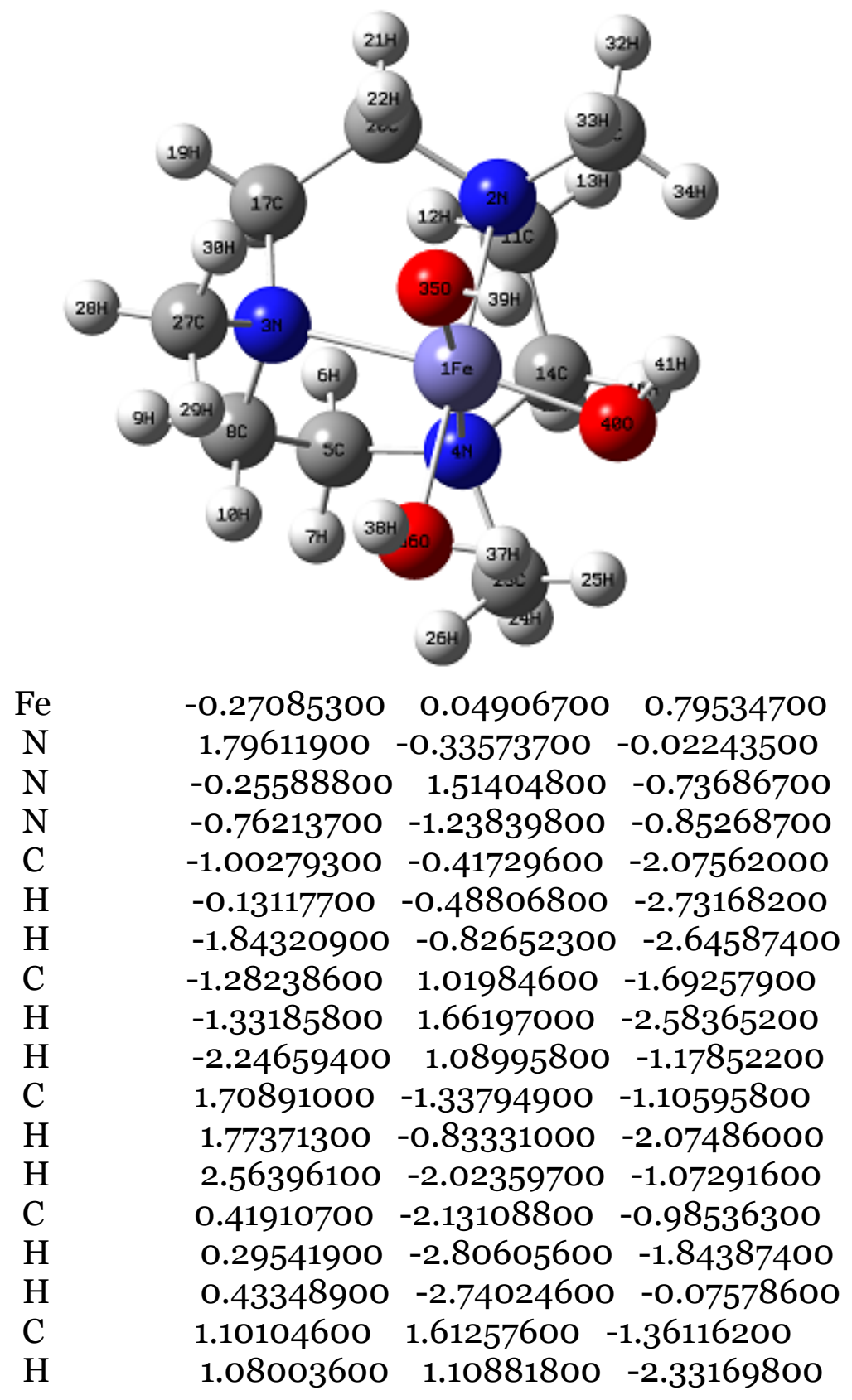




$\begin{array}{lccc}\mathrm{H} & 1.33377600 & 2.66179100 & -1.57145500 \\ \mathrm{C} & 2.16948600 & 1.01973300 & -0.46455400 \\ \mathrm{H} & 3.14167600 & 1.03234200 & -0.98073100 \\ \mathrm{H} & 2.26330400 & 1.62275800 & 0.44457500 \\ \mathrm{C} & -1.95383000 & -2.04950500 & -0.52977600 \\ \mathrm{H} & -2.12444400 & -2.80728900 & -1.30425600 \\ \mathrm{H} & -1.79377300 & -2.53866400 & 0.43266500 \\ \mathrm{H} & -2.82781400 & -1.40002000 & -0.46468000 \\ \mathrm{C} & -0.65866300 & 2.82666000 & -0.19493500 \\ \mathrm{H} & -0.73692800 & 3.56704800 & -1.00028500 \\ \mathrm{H} & -1.62706200 & 2.73356200 & 0.29944900 \\ \mathrm{H} & 0.07058400 & 3.14866700 & 0.54812200 \\ \mathrm{C} & 2.65943600 & -0.79174700 & 1.07208000 \\ \mathrm{H} & 3.71180800 & -0.85274000 & 0.76303700 \\ \mathrm{H} & 2.57367100 & -0.09345400 & 1.90864200 \\ \mathrm{H} & 2.33535300 & -1.78174800 & 1.40240700 \\ \mathrm{O} & 0.20810100 & 1.29205800 & 2.02913500 \\ \mathrm{O} & -2.29604900 & 0.23970900 & 1.22966900 \\ \mathrm{H} & -2.19824300 & -0.49935100 & 1.85186100 \\ \mathrm{H} & -2.40209300 & 1.01303300 & 1.79673500 \\ \mathrm{H} & 0.19175300 & 0.95536600 & 2.93309300 \\ \mathrm{O} & -0.42822200 & -1.38615000 & 1.92936600 \\ \mathrm{H} & 0.25764600 & -1.43169100 & 2.60641600 \\ & & & \end{array}$

$\left[\mathrm{Fe}\left(\mathrm{Me}_{3} \operatorname{tacn}\right)\left(\mathrm{OH}_{2}\right)(\mathrm{OH})(\mathrm{O})\right]^{+}$

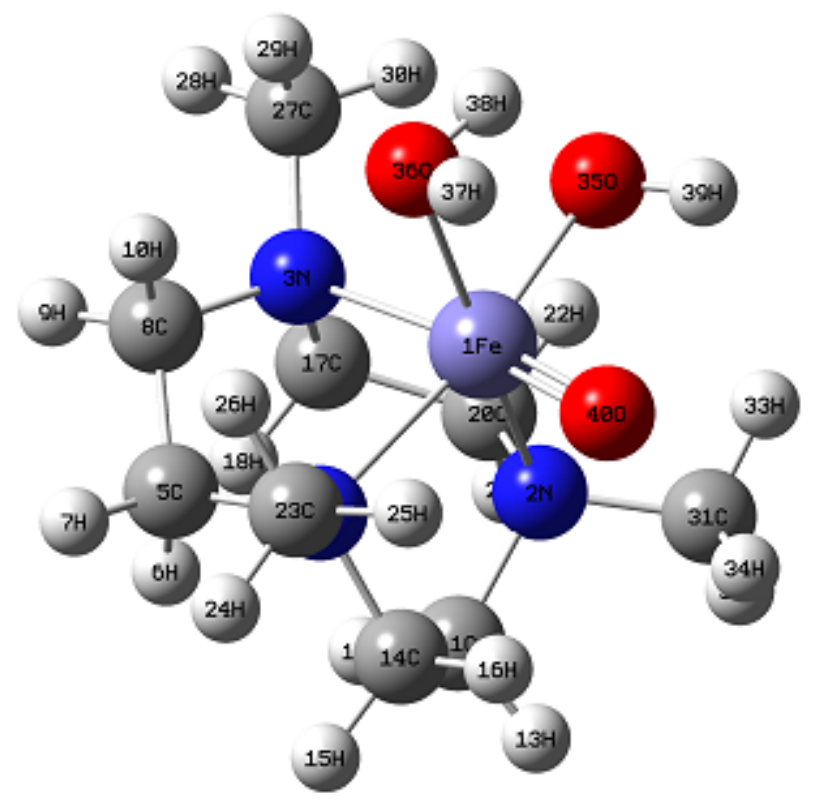

$\begin{array}{cccc}\mathrm{Fe} & 0.03366200 & -0.06161500 & 0.80014200 \\ \mathrm{~N} & -1.25257200 & -1.08565500 & -0.36258200 \\ \mathrm{~N} & 1.42633100 & -0.36634800 & -0.83757700 \\ \mathrm{~N} & -0.50273000 & 1.60768400 & -0.36600800 \\ \mathrm{C} & 0.35885600 & 1.72206200 & -1.57750000 \\ \mathrm{H} & -0.17733200 & 1.30866800 & -2.43599000 \\ \mathrm{H} & 0.53943500 & 2.77618200 & -1.81362400 \\ \mathrm{C} & 1.67033100 & 0.99975700 & -1.35702100 \\ \mathrm{H} & 2.26352900 & 0.97897200 & -2.28246600 \\ \mathrm{H} & 2.26492200 & 1.51636600 & -0.59602200\end{array}$




$\begin{array}{lccc}\mathrm{C} & -2.00984000 & -0.12504300 & -1.23486300 \\ \mathrm{H} & -1.61006000 & -0.18890200 & -2.25031700 \\ \mathrm{H} & -3.05646900 & -0.43712400 & -1.29719800 \\ \mathrm{C} & -1.91445300 & 1.28589500 & -0.69468800 \\ \mathrm{H} & -2.32784400 & 2.00508900 & -1.41521800 \\ \mathrm{H} & -2.47329000 & 1.37338500 & 0.24066700 \\ \mathrm{C} & 0.73663200 & -1.24765800 & -1.82423400 \\ \mathrm{H} & 0.36443400 & -0.63851600 & -2.65295000 \\ \mathrm{H} & 1.44864100 & -1.95313100 & -2.26570300 \\ \mathrm{C} & -0.38491200 & -2.00874100 & -1.15076800 \\ \mathrm{H} & -0.98972300 & -2.55757100 & -1.88472700 \\ \mathrm{H} & 0.02501700 & -2.71885000 & -0.42798300 \\ \mathrm{C} & -0.43208100 & 2.84180900 & 0.43883100 \\ \mathrm{H} & -0.85989700 & 3.69045700 & -0.10839700 \\ \mathrm{H} & -0.98147400 & 2.68459300 & 1.36853200 \\ \mathrm{H} & 0.61299400 & 3.06222000 & 0.66823600 \\ \mathrm{C} & 2.69795200 & -0.98152600 & -0.40997700 \\ \mathrm{H} & 3.37433600 & -1.10173800 & -1.26498400 \\ \mathrm{H} & 3.17939100 & -0.34084200 & 0.33048500 \\ \mathrm{H} & 2.49203500 & -1.94916700 & 0.04805000 \\ \mathrm{C} & -2.18757400 & -1.87065000 & 0.47361900 \\ \mathrm{H} & -2.84298800 & -2.47535000 & -0.16347100 \\ \mathrm{H} & -1.60860800 & -2.52466700 & 1.12562900 \\ \mathrm{H} & -2.77316100 & -1.18701000 & 1.08831100 \\ \mathrm{O} & 0.76245000 & -1.60360400 & 1.59158000 \\ \mathrm{O} & 1.59004400 & 0.78919900 & 1.85899900 \\ \mathrm{H} & 1.17750200 & 1.26442400 & 2.59477300 \\ \mathrm{H} & 1.77769200 & -0.11352500 & 2.21420500 \\ \mathrm{H} & 0.19336500 & -1.85038100 & 2.33052700 \\ \mathrm{O} & -1.07678400 & 0.33855600 & 1.92800900\end{array}$

TS

$\mathrm{Fe}$

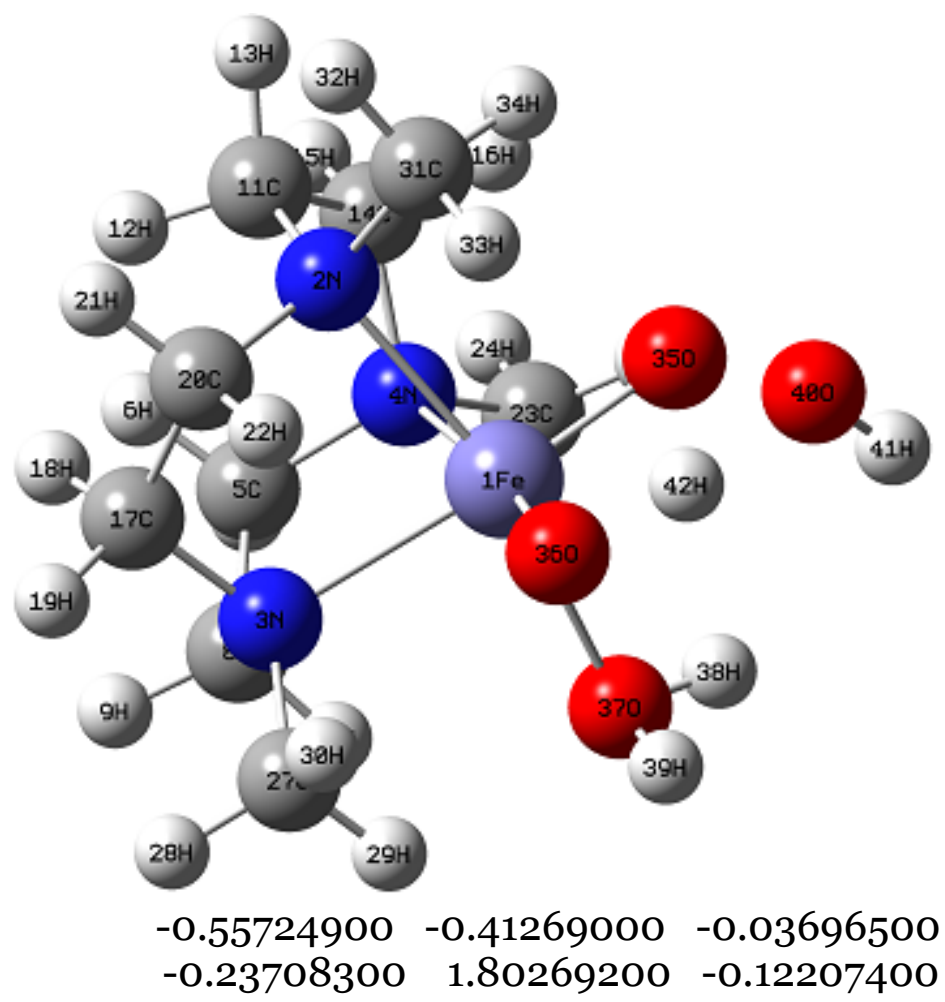




$\begin{array}{lccc}\mathrm{N} & 1.21557500 & -0.35891700 & -1.28401700 \\ \mathrm{~N} & 0.97186000 & -0.20090900 & 1.48981300 \\ \mathrm{C} & 2.34264900 & -0.29549900 & 0.90112000 \\ \mathrm{H} & 2.76662600 & 0.70766700 & 0.83011900 \\ \mathrm{H} & 2.99960500 & -0.85444700 & 1.57361700 \\ \mathrm{C} & 2.28788700 & -0.96630200 & -0.45143000 \\ \mathrm{H} & 3.25827600 & -0.89676700 & -0.95989700 \\ \mathrm{H} & 2.04228100 & -2.02602700 & -0.34403900 \\ \mathrm{C} & 0.66022600 & 2.20974200 & 0.98476700 \\ \mathrm{H} & 1.65958100 & 2.40119500 & 0.58679800 \\ \mathrm{H} & 0.32359300 & 3.15681900 & 1.41895600 \\ \mathrm{C} & 0.69384800 & 1.14399900 & 2.06297000 \\ \mathrm{H} & 1.44068600 & 1.39110700 & 2.82829600 \\ \mathrm{H} & -0.28223400 & 1.07164100 & 2.55333600 \\ \mathrm{C} & 1.53535400 & 1.05741400 & -1.65410800 \\ \mathrm{H} & 2.38183900 & 1.39325700 & -1.05109700 \\ \mathrm{H} & 1.87211200 & 1.09439600 & -2.69413600 \\ \mathrm{C} & 0.34484800 & 1.97263900 & -1.46640300 \\ \mathrm{H} & 0.64233200 & 3.01518400 & -1.64752800 \\ \mathrm{H} & -0.44469500 & 1.71853900 & -2.18068300 \\ \mathrm{C} & 0.78763700 & -1.22841400 & 2.53339200 \\ \mathrm{H} & 1.41797700 & -1.01068500 & 3.40310200 \\ \mathrm{H} & -0.26119500 & -1.24511100 & 2.83718600 \\ \mathrm{H} & 1.07026600 & -2.20488400 & 2.13603600 \\ \mathrm{C} & 1.00947700 & -1.15936900 & -2.50640200 \\ \mathrm{H} & 1.91380500 & -1.14978300 & -3.12575200 \\ \mathrm{H} & 0.78176400 & -2.19108700 & -2.23567100 \\ \mathrm{H} & 0.17006700 & -0.74517000 & -3.06503300 \\ \mathrm{C} & -1.55080200 & 2.45150800 & -0.02078000 \\ \mathrm{H} & -1.46668700 & 3.53562200 & -0.16959600 \\ \mathrm{H} & -2.21721700 & 2.02847900 & -0.77498600 \\ \mathrm{H} & -1.97619300 & 2.25063700 & 0.96381900 \\ \mathrm{O} & -1.76034300 & -0.41677600 & 1.12818100 \\ \mathrm{O} & -1.62426600 & -0.49660100 & -1.38951700 \\ \mathrm{O} & -0.26591500 & -2.67729500 & -0.01292400 \\ \mathrm{H} & -0.68596300 & -3.03492400 & 0.78069300 \\ \mathrm{H} & -0.85995300 & -2.94342400 & -0.72857500 \\ \mathrm{O} & -3.60560400 & -0.49003200 & -0.02317400 \\ \mathrm{H} & -3.66815300 & -1.37274000 & 0.36526900 \\ \mathrm{H} & -2.66024100 & -0.52829700 & -0.84448900\end{array}$

$\left[\mathrm{Fe}\left(\mathrm{Me}_{3} \mathrm{tacn}\right)(\mathrm{Cl})(\mathrm{O})_{2}\right] \cdot \mathrm{H}_{2} \mathrm{O}$ 


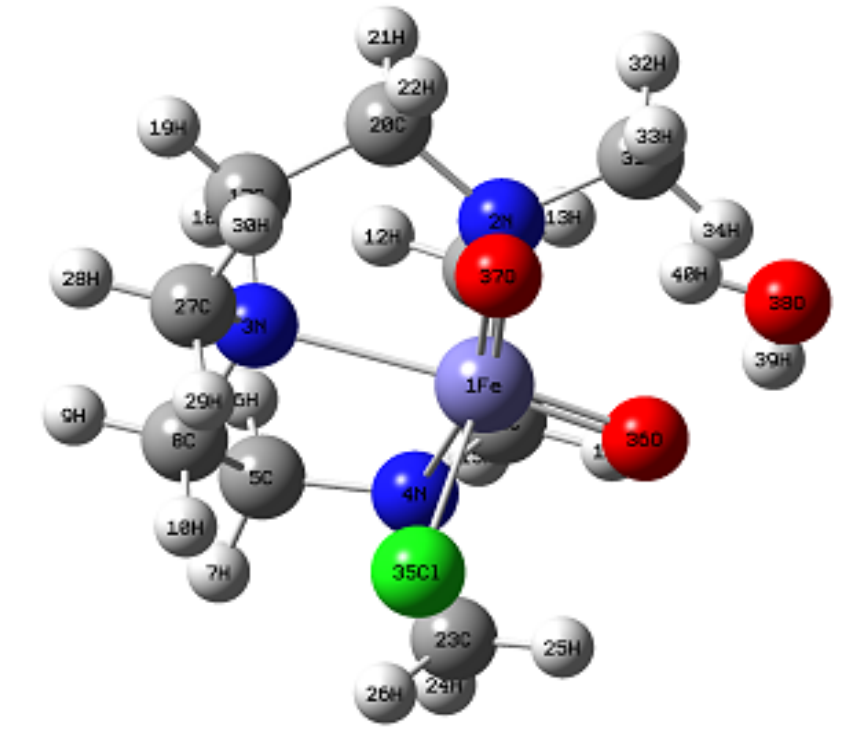

$$
\begin{aligned}
& \begin{array}{llll}
\mathrm{Fe} & -0.52973900 & -0.39631800 & 0.00958700
\end{array} \\
& \begin{array}{llll}
\mathrm{N} & -0.50222300 & 1.72064300 & -0.04788700
\end{array} \\
& \mathrm{~N} \quad \begin{array}{llll}
1.21602600 & -0.06196900 & -1.40067300
\end{array} \\
& \begin{array}{lllll}
\mathrm{N} & & 1.22218100 & 0.00955600 & 1.41763700
\end{array} \\
& \begin{array}{lllll}
\mathrm{C} & 2.50970000 & 0.14914100 & 0.68770900
\end{array} \\
& \begin{array}{llll}
\mathrm{H} & 2.76542900 & 1.21095000 & 0.60919100
\end{array} \\
& \mathrm{H} \quad 3.32142700 \quad-0.30977800 \quad 1.26533200 \\
& \begin{array}{llll}
\mathrm{C} & 2.42762800 & -0.49862000 & -0.67803600
\end{array} \\
& \mathrm{H} \quad 3.33560400 \quad-0.27819700 \quad-1.26171800 \\
& \mathrm{H} \quad 2.34816800 \quad-1.58631000 \quad-0.57251600 \\
& \begin{array}{llll}
\mathrm{C} & 0.41654100 & 2.29273300 & 0.98485000
\end{array} \\
& \begin{array}{llll}
\mathrm{H} & 1.30937600 & 2.67332800 & 0.48112300
\end{array} \\
& \mathrm{H} \quad \begin{array}{llll}
\mathrm{H} & -0.06471300 & 3.15918700 & 1.45205000
\end{array} \\
& \begin{array}{llll}
\mathrm{C} & 0.77414100 & 1.27090600 & 2.03787500
\end{array} \\
& \begin{array}{llll}
\mathrm{H} & 1.54412800 & 1.67844600 & 2.71183700
\end{array} \\
& \mathrm{H} \quad \begin{array}{llll}
0.10923600 & 1.02337200 & 2.63562300
\end{array} \\
& \begin{array}{lllll}
\mathrm{C} & & 1.25027900 & 1.38366500 & -1.74843900
\end{array} \\
& \mathrm{H} \quad 2.07248900 \quad 1.86426400 \quad-1.20980500 \\
& \mathrm{H} \quad \begin{array}{llll}
\mathrm{H} & 1.47646300 & 1.51035900 & -2.81427800
\end{array} \\
& \begin{array}{llll}
\mathrm{C} & -0.06820000 & 2.05151200 & -1.42951500
\end{array} \\
& \mathrm{H} \quad \begin{array}{llll}
\mathrm{C} & 0.01177800 & 3.14270300 & -1.54021000
\end{array} \\
& \mathrm{H} \quad-\begin{array}{llll}
0.85975800 & 1.69038700 & -2.09259200
\end{array} \\
& \begin{array}{llll}
\mathrm{C} & 1.31872200 & -1.02942700 & 2.45065800
\end{array} \\
& \mathrm{H} \quad \begin{array}{llll}
1.99013200 & -0.70778800 & 3.26093800
\end{array} \\
& \mathrm{H} \quad 0.31850900 \quad-1.21800100 \quad 2.85064000 \\
& \mathrm{H} \quad \begin{array}{llll}
\mathrm{H} & 1.70058100 & -1.95078700 & 2.00588100
\end{array} \\
& \text { C } \quad \begin{array}{llll}
\mathrm{H} & 1.02904800 & -0.86148700 & -2.61851400
\end{array} \\
& \mathrm{H} \quad \begin{array}{llll}
\mathrm{C} & 1.87141800 & -0.71620000 & -3.31073900
\end{array} \\
& \mathrm{H} \quad \begin{array}{llll}
0.95299700 & -1.91710800 & -2.34630800
\end{array} \\
& \mathrm{H} \quad \begin{array}{llll}
\mathrm{H} & 0.09600900 & -0.55626000 & -3.09845100
\end{array} \\
& \begin{array}{llll}
\text { C } & -1.88199000 & 2.18559800 & 0.17787300
\end{array} \\
& \mathrm{H} \quad \begin{array}{llll}
\mathrm{H} & -1.94218200 & 3.26834300 & 0.00559300
\end{array}
\end{aligned}
$$

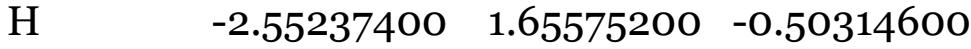

$$
\begin{aligned}
& \mathrm{H} \quad-2.16623200 \quad 1.95459000 \quad 1.20683700 \\
& \mathrm{Cl} \quad-0.05454000 \quad-2.66925200 \quad 0.00868600 \\
& \begin{array}{llll}
\mathrm{O} & -1.49315300 & -0.41057200 & 1.35102400
\end{array} \\
& \begin{array}{llll}
\mathrm{O} & -1.54019300 & -0.42606100 & -1.29678400
\end{array}
\end{aligned}
$$




$\begin{array}{llll}\mathrm{O} & -3.99322900 & -0.66794600 & 0.04825400 \\ \mathrm{H} & -3.34941600 & -0.57241600 & 0.77521800 \\ \mathrm{H} & -3.36470600 & -0.64240400 & -0.69766800\end{array}$

\section{[Fe( $\left.\left.\mathrm{Me}_{3} \mathrm{tacn}\right)(\mathrm{Cl})(\mathrm{OH})(\mathrm{OOH})\right]$}

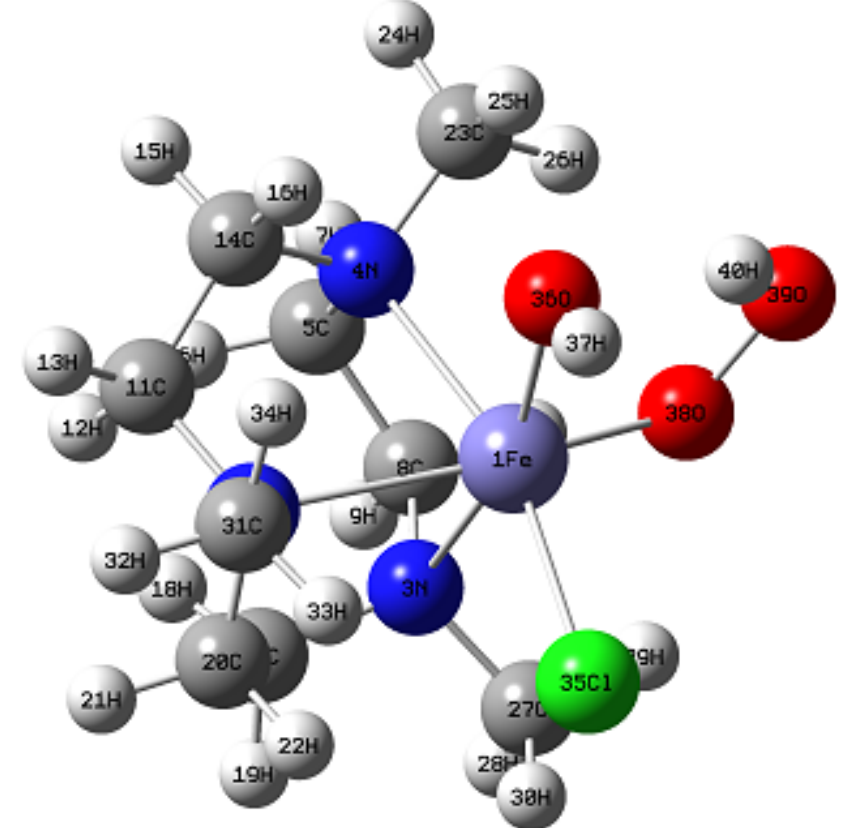

$\begin{array}{cccc}\mathrm{Fe} & -0.24115900 & -0.69151800 & -0.32083300 \\ \mathrm{~N} & 1.57912600 & 0.73280500 & -0.79093500 \\ \mathrm{~N} & 0.40206900 & 0.12178900 & 1.75183800 \\ \mathrm{~N} & -1.12223800 & 1.49498100 & -0.24127000 \\ \mathrm{C} & -0.98941800 & 2.07111000 & 1.11171500 \\ \mathrm{H} & -0.18893700 & 2.82054300 & 1.10872800 \\ \mathrm{H} & -1.90526200 & 2.61562100 & 1.38645800 \\ \mathrm{C} & -0.71389700 & 0.99893200 & 2.15212000 \\ \mathrm{H} & -0.51320200 & 1.47035000 & 3.13197700 \\ \mathrm{H} & -1.59126700 & 0.35093800 & 2.25884000 \\ \mathrm{C} & 1.14070700 & 2.13036800 & -0.99147100 \\ \mathrm{H} & 1.40558300 & 2.72180300 & -0.10677100 \\ \mathrm{H} & 1.69148800 & 2.58616900 & -1.82767500 \\ \mathrm{C} & -0.34972200 & 2.21021600 & -1.26483600 \\ \mathrm{H} & -0.66185600 & 3.26854500 & -1.34388100 \\ \mathrm{H} & -0.58205100 & 1.71576900 & -2.21692000 \\ \mathrm{C} & 1.69652200 & 0.84106500 & 1.68131700 \\ \mathrm{H} & 1.51178600 & 1.91669600 & 1.79507400 \\ \mathrm{H} & 2.33151100 & 0.55666800 & 2.53273300 \\ \mathrm{C} & 2.44432100 & 0.56048100 & 0.38654600 \\ \mathrm{H} & 3.33845100 & 1.20876500 & 0.32814200 \\ \mathrm{H} & 2.78064900 & -0.48307200 & 0.37084100 \\ \mathrm{C} & -2.53252800 & 1.39854200 & -0.63226900 \\ \mathrm{H} & -2.98488400 & 2.40092700 & -0.72704300 \\ \mathrm{H} & -2.59958800 & 0.87156200 & -1.58836500 \\ \mathrm{H} & -3.07668000 & 0.81670400 & 0.11627800 \\ \mathrm{C} & 0.48421400 & -1.03088400 & 2.66145300 \\ \mathrm{H} & 0.63721800 & -0.70105200 & 3.70315800 \\ \mathrm{H} & -0.44206600 & -1.60918600 & 2.58847700 \\ \mathrm{H} & 1.31127100 & -1.67612500 & 2.35333500 \\ & & & \\ & & & \\ & & & \end{array}$




$\begin{array}{llll}\mathrm{C} & 2.23324700 & 0.22944300 & -2.00352100 \\ \mathrm{H} & 3.15061800 & 0.79956800 & -2.23042500 \\ \mathrm{H} & 2.47883900 & -0.82770000 & -1.86832900 \\ \mathrm{H} & 1.53795800 & 0.32007000 & -2.84497200 \\ \mathrm{Cl} & 1.21356100 & -2.50485500 & -0.30201500 \\ \mathrm{O} & -0.99745500 & -0.76725500 & -2.07479900 \\ \mathrm{H} & -0.58771700 & -1.47967700 & -2.58835800 \\ \mathrm{O} & -1.79779700 & -1.31560400 & 0.62107000 \\ \mathrm{O} & -2.88117900 & -1.71909200 & -0.21927100 \\ \mathrm{H} & -2.48420700 & -1.58894600 & -1.11513900\end{array}$

\section{[Fe( $\left.\left.\mathrm{Me}_{3} \mathrm{tacn}\right)(\mathrm{Cl})(\mathrm{OH})(\mathrm{OO})\right]$}

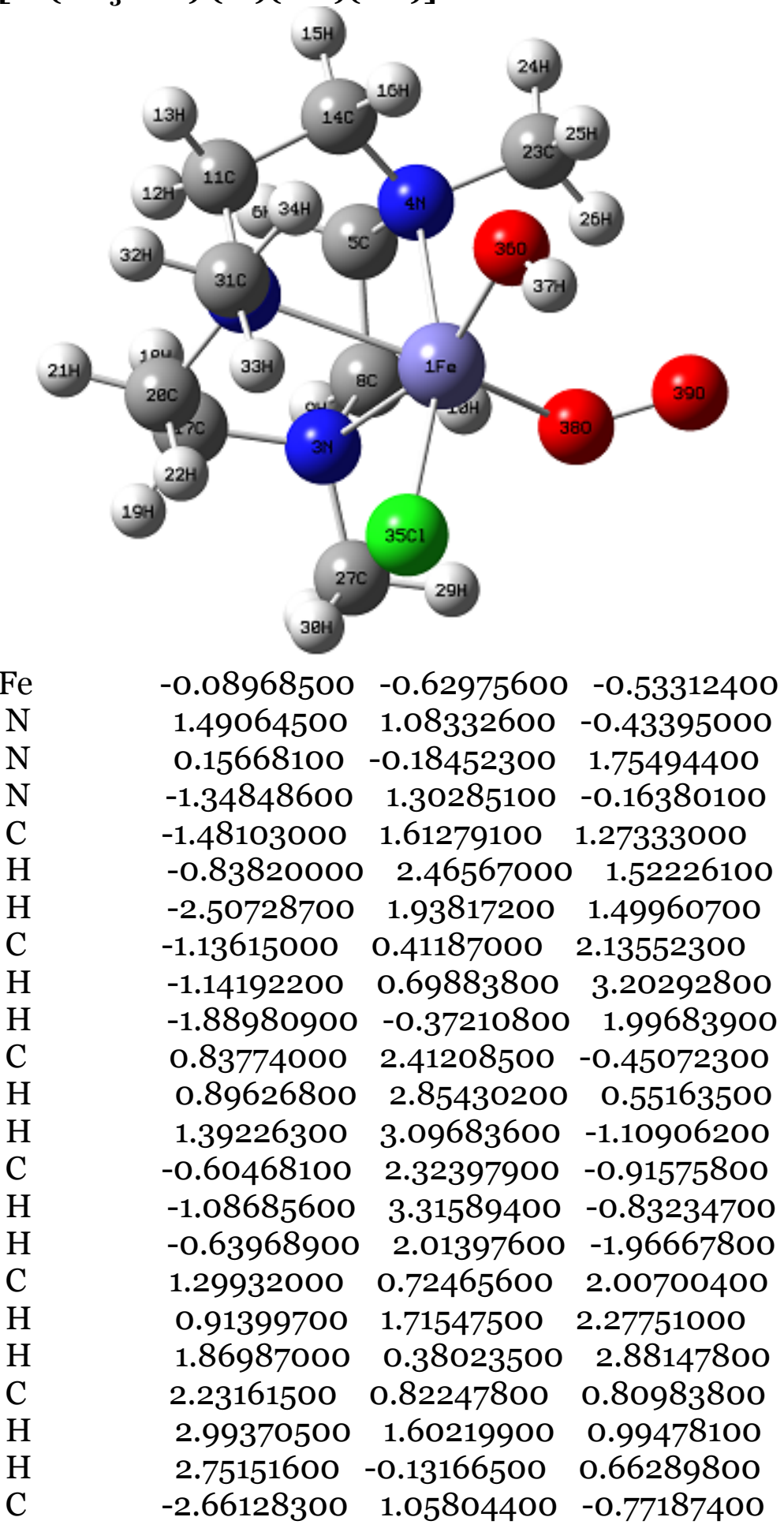




\begin{tabular}{|c|c|c|c|}
\hline $\mathrm{H}$ & -3.28128400 & 1.97078400 & -0.75418900 \\
\hline $\mathrm{H}$ & -2.51626000 & 0.72155100 & -1.80161900 \\
\hline $\mathrm{H}$ & -3.17190500 & 0.25719100 & -0.22931700 \\
\hline $\mathrm{C}$ & 0.33974900 & -1.46451300 & 2.45315200 \\
\hline $\mathrm{H}$ & 0.28153400 & -1.32964800 & 3.54649500 \\
\hline $\mathrm{H}$ & -0.43495900 & -2.16564800 & 2.12761300 \\
\hline $\mathrm{H}$ & 1.30958000 & -1.88865800 & 2.18172200 \\
\hline $\mathrm{C}$ & 2.36299800 & 0.92924600 & -1.60410600 \\
\hline $\mathrm{H}$ & 3.19483200 & 1.65383500 & -1.57658800 \\
\hline $\mathrm{H}$ & 2.75865400 & -0.09033900 & -1.62345600 \\
\hline $\mathrm{H}$ & 1.76887700 & 1.08434600 & -2.50929500 \\
\hline $\mathrm{Cl}$ & 1.60701200 & -2.21863500 & -0.53278500 \\
\hline $\mathrm{O}$ & -0.44777000 & -0.30401900 & -2.33320100 \\
\hline $\mathrm{H}$ & -0.16856900 & -1.04682500 & -2.88950100 \\
\hline $\mathrm{O}$ & -1.55425400 & -1.71368000 & 0.06949900 \\
\hline $\mathrm{O}$ & -2.58088900 & -2.11347200 & -0.60552400 \\
\hline
\end{tabular}

\section{$\left[\mathrm{Fe}\left(\mathrm{Me}_{3}\right.\right.$ tacn $\left.)(\mathrm{Cl})(\mathrm{OH})\left(\mathrm{H}_{2} \mathrm{O}\right)\right]$}

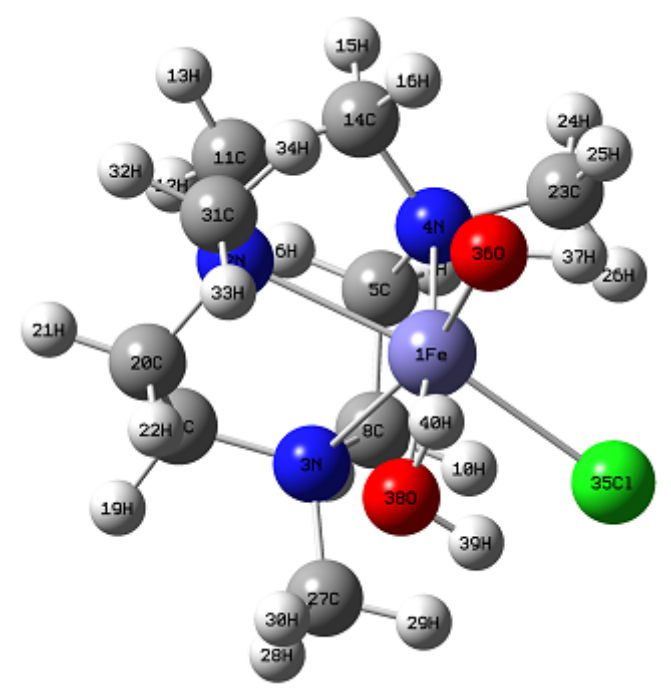

$\begin{array}{cccl}\mathrm{Fe} & -0.47671000 & -0.41590300 & -0.49224100 \\ \mathrm{~N} & 1.79337000 & -0.75977900 & -0.08616300 \\ \mathrm{~N} & -0.19517300 & 0.19611400 & 1.72927300 \\ \mathrm{~N} & 0.35006600 & 1.68474900 & -0.66927800 \\ \mathrm{C} & 0.38597400 & 2.35445000 & 0.64621800 \\ \mathrm{H} & 1.42440500 & 2.40704000 & 0.99699200 \\ \mathrm{H} & 0.05045500 & 3.39831300 & 0.55374400 \\ \mathrm{C} & -0.49334900 & 1.63867700 & 1.66410500 \\ \mathrm{H} & -0.38430800 & 2.11761900 & 2.65522700 \\ \mathrm{H} & -1.54636100 & 1.71848300 & 1.36100700 \\ \mathrm{C} & 2.51598400 & 0.48731100 & -0.40665000 \\ \mathrm{H} & 2.82325700 & 0.97958900 & 0.52420900 \\ \mathrm{H} & 3.45015500 & 0.26173500 & -0.94408400 \\ \mathrm{C} & 1.67536500 & 1.43089300 & -1.25565200 \\ \mathrm{H} & 2.23368900 & 2.37109200 & -1.42784600 \\ \mathrm{H} & 1.49005600 & 0.96588500 & -2.23347600 \\ \mathrm{C} & 1.17104800 & -0.09625300 & 2.20871300 \\ \mathrm{H} & 1.75257700 & 0.83295200 & 2.23464700 \\ \mathrm{H} & 1.14331900 & -0.45796000 & 3.24772000\end{array}$




$\begin{array}{lccc}\mathrm{C} & 1.85774700 & -1.12911800 & 1.33144300 \\ \mathrm{H} & 2.89958400 & -1.27805900 & 1.67618700 \\ \mathrm{H} & 1.34109700 & -2.09393900 & 1.42487200 \\ \mathrm{C} & -0.51703300 & 2.39706400 & -1.60940000 \\ \mathrm{H} & -0.12619200 & 3.40340500 & -1.84438400 \\ \mathrm{H} & -0.59560100 & 1.81549100 & -2.53419800 \\ \mathrm{H} & -1.52196100 & 2.47695200 & -1.18287800 \\ \mathrm{C} & -1.18828100 & -0.50800600 & 2.54756900 \\ \mathrm{H} & -1.16679000 & -0.15879500 & 3.59453700 \\ \mathrm{H} & -2.18263300 & -0.34545400 & 2.12108200 \\ \mathrm{H} & -0.97456600 & -1.58187500 & 2.52380900 \\ \mathrm{C} & 2.23690100 & -1.86204900 & -0.94084300 \\ \mathrm{H} & 3.30534700 & -2.09365100 & -0.78105000 \\ \mathrm{H} & 1.64079100 & -2.75248000 & -0.71832700 \\ \mathrm{H} & 2.05482700 & -1.60407000 & -1.98748000 \\ \mathrm{Cl} & -2.84955200 & 0.06943700 & -0.38048500 \\ \mathrm{O} & -0.28795800 & -1.19727600 & -2.24369800 \\ \mathrm{H} & -1.06962500 & -1.00117800 & -2.77998500 \\ \mathrm{O} & -1.16812400 & -2.65971200 & -0.02564100 \\ \mathrm{H} & -2.09583200 & -2.35695000 & -0.00037100 \\ \mathrm{H} & -0.96415400 & -2.66058000 & -0.98858700 \\ {\left[\mathbf{F e}\left(\mathbf{M e}_{3} \mathbf{t a c n}\right)(\mathbf{C l})(\mathbf{O H})(\mathbf{O H})\right]} & & \end{array}$

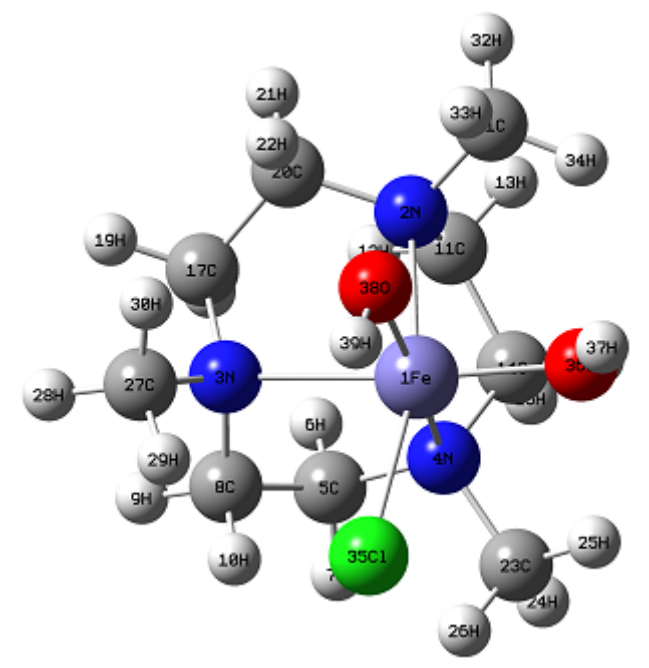

$\begin{array}{cccc}\mathrm{Fe} & 0.32988700 & -0.22826900 & -0.73644400 \\ \mathrm{~N} & -1.90220300 & 0.16055800 & -0.34964100 \\ \mathrm{~N} & 0.33016100 & 1.53552000 & 0.76064100 \\ \mathrm{~N} & 0.10095700 & -1.26808300 & 1.11320700 \\ \mathrm{C} & 0.29219900 & -0.37899200 & 2.29668700 \\ \mathrm{H} & -0.68831400 & -0.18143900 & 2.74760300 \\ \mathrm{H} & 0.87700500 & -0.90662600 & 3.06205700 \\ \mathrm{C} & 0.98472000 & 0.91669900 & 1.92343300 \\ \mathrm{H} & 1.00177300 & 1.60039100 & 2.79236700 \\ \mathrm{H} & 2.01903000 & 0.71285600 & 1.62087600 \\ \mathrm{C} & -2.30635500 & -0.70370000 & 0.77625400 \\ \mathrm{H} & -2.44962100 & -0.08871500 & 1.67329900 \\ \mathrm{H} & -3.28447300 & -1.16684800 & 0.57549900 \\ \mathrm{C} & -1.28313000 & -1.80134200 & 1.03262000 \\ \mathrm{H} & -1.53448200 & -2.34739100 & 1.95878400 \\ \mathrm{H} & -1.27867800 & -2.51200800 & 0.19930900\end{array}$




$\begin{array}{lccc}\mathrm{C} & -1.04428600 & 2.00012300 & 1.04058400 \\ \mathrm{H} & -1.37592200 & 1.59326300 & 2.00364300 \\ \mathrm{H} & -1.05858500 & 3.09392600 & 1.15766100 \\ \mathrm{C} & -1.99608500 & 1.59485300 & -0.06987100 \\ \mathrm{H} & -3.02811600 & 1.90093900 & 0.18631900 \\ \mathrm{H} & -1.71435600 & 2.10429000 & -1.00051100 \\ \mathrm{C} & 1.06094600 & -2.38748300 & 1.15152300 \\ \mathrm{H} & 0.86275600 & -3.03105300 & 2.02370900 \\ \mathrm{H} & 0.96278500 & -2.95596600 & 0.22327100 \\ \mathrm{H} & 2.07488000 & -1.98131900 & 1.20031300 \\ \mathrm{C} & 1.13467300 & 2.63865800 & 0.22275000 \\ \mathrm{H} & 1.24595600 & 3.44390900 & 0.96883900 \\ \mathrm{H} & 2.11748100 & 2.25640200 & -0.06767500 \\ \mathrm{H} & 0.63979000 & 3.03217900 & -0.67038600 \\ \mathrm{C} & -2.61331900 & -0.18068500 & -1.58208400 \\ \mathrm{H} & -3.68692200 & 0.06806500 & -1.50856900 \\ \mathrm{H} & -2.16031700 & 0.36914300 & -2.41281600 \\ \mathrm{H} & -2.48811800 & -1.24852800 & -1.77804100 \\ \mathrm{Cl} & 2.74236900 & -0.15110300 & -0.72923100 \\ \mathrm{O} & -0.09673200 & -1.84302300 & -1.56599000 \\ \mathrm{H} & 0.14186900 & -1.77221600 & -2.50208600 \\ \mathrm{O} & 0.26396600 & 0.88103900 & -2.17125000 \\ \mathrm{H} & 1.19078000 & 1.03160100 & -2.43189600 \\ & & & \end{array}$

\section{$\left[\mathrm{Fe}\left(\mathrm{Me}_{3} \mathrm{tacn}\right)(\mathrm{Cl})(\mathrm{O})(\mathrm{OH})\right]$}

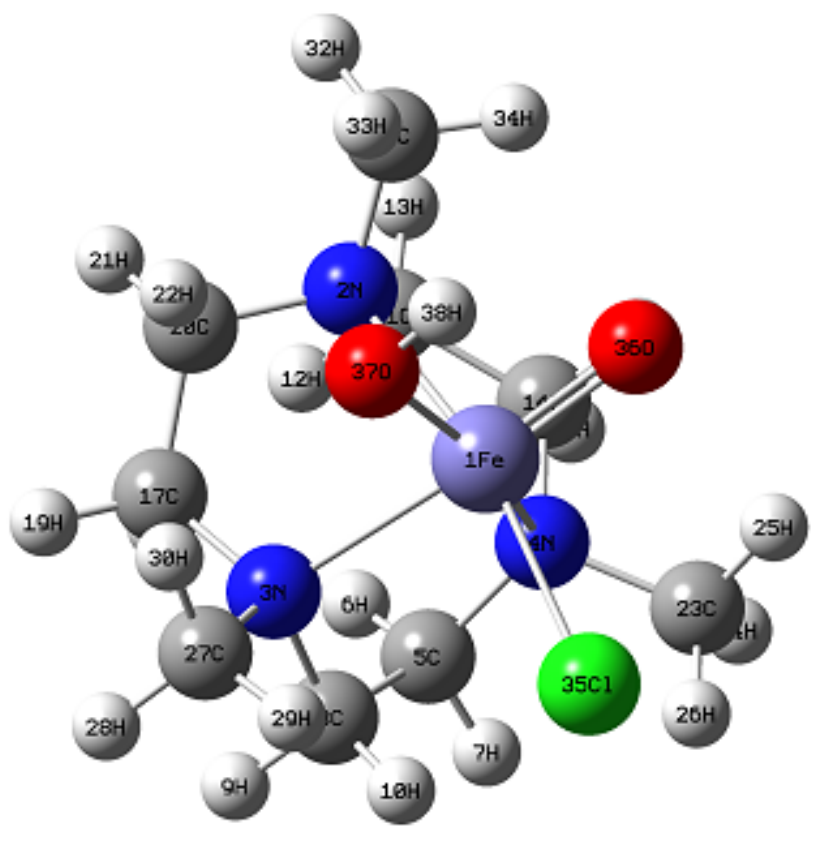

$\begin{array}{cccc}\mathrm{Fe} & -0.34281000 & -0.13380100 & -0.84949400 \\ \mathrm{~N} & 1.94063500 & -0.06196400 & -0.22274600 \\ \mathrm{~N} & -0.30494200 & -1.18905900 & 1.14202300 \\ \mathrm{~N} & -0.22562800 & 1.59348900 & 0.62123700 \\ \mathrm{C} & -0.43593900 & 1.12352900 & 2.01473200 \\ \mathrm{H} & 0.51975300 & 1.15952900 & 2.55215100 \\ \mathrm{H} & -1.10415400 & 1.81523900 & 2.54602100 \\ \mathrm{C} & -1.02655800 & -0.27656800 & 2.05405400\end{array}$ 


\begin{tabular}{|c|c|c|c|}
\hline $\mathrm{H}$ & -1.00982500 & -0.66475200 & 3.08823000 \\
\hline $\mathrm{H}$ & -2.06591400 & -0.25471500 & 1.70968100 \\
\hline $\mathrm{C}$ & 2.21352900 & 1.13416600 & 0.58548400 \\
\hline $\mathrm{H}$ & 2.30693000 & 0.84920300 & 1.64040400 \\
\hline $\mathrm{H}$ & 3.18186700 & 1.57819400 & 0.30786000 \\
\hline $\mathrm{C}$ & 1.11603000 & 2.16564200 & 0.40136100 \\
\hline $\mathrm{H}$ & 1.28594300 & 3.02866200 & 1.06951700 \\
\hline $\mathrm{H}$ & 1.11854300 & 2.52956000 & -0.63370300 \\
\hline $\mathrm{C}$ & 1.08211800 & -1.45067600 & 1.61158900 \\
\hline $\mathrm{H}$ & 1.31691600 & -0.75369800 & 2.42553300 \\
\hline $\mathrm{H}$ & 1.13971000 & -2.45539400 & 2.05348900 \\
\hline $\mathrm{C}$ & 2.10072800 & -1.32918800 & 0.49625300 \\
\hline $\mathrm{H}$ & 3.12109600 & -1.44234100 & 0.90917000 \\
\hline $\mathrm{H}$ & 1.93726400 & -2.12025900 & -0.24410000 \\
\hline $\mathrm{C}$ & -1.23900200 & 2.58938500 & 0.23763500 \\
\hline $\mathrm{H}$ & -1.09410000 & 3.52387700 & 0.80493300 \\
\hline $\mathrm{H}$ & -1.15099000 & 2.78081700 & -0.83586100 \\
\hline $\mathrm{H}$ & -2.23507100 & 2.18341600 & 0.42482300 \\
\hline $\mathrm{C}$ & -1.03362700 & -2.46079100 & 1.00089000 \\
\hline $\mathrm{H}$ & -1.10932500 & -2.97257900 & 1.97423700 \\
\hline $\mathrm{H}$ & -2.03080100 & -2.25643700 & 0.60217300 \\
\hline $\mathrm{H}$ & -0.50210800 & -3.09035400 & 0.28276200 \\
\hline $\mathrm{C}$ & 2.70161900 & -0.04847800 & -1.46996500 \\
\hline $\mathrm{H}$ & 3.78777500 & -0.09906700 & -1.27818900 \\
\hline $\mathrm{H}$ & 2.39308700 & -0.90273500 & -2.07816900 \\
\hline $\mathrm{H}$ & 2.46400900 & 0.86342100 & -2.02584200 \\
\hline $\mathrm{Cl}$ & -2.71270100 & -0.12471900 & -0.76299400 \\
\hline $\mathrm{O}$ & -0.07952700 & 0.86717300 & -2.11478800 \\
\hline $\mathrm{O}$ & 0.07914100 & -1.75890500 & -1.63714700 \\
\hline $\mathrm{H}$ & 0.01762000 & -1.64606800 & -2.59769100 \\
\hline
\end{tabular}

TS Cl

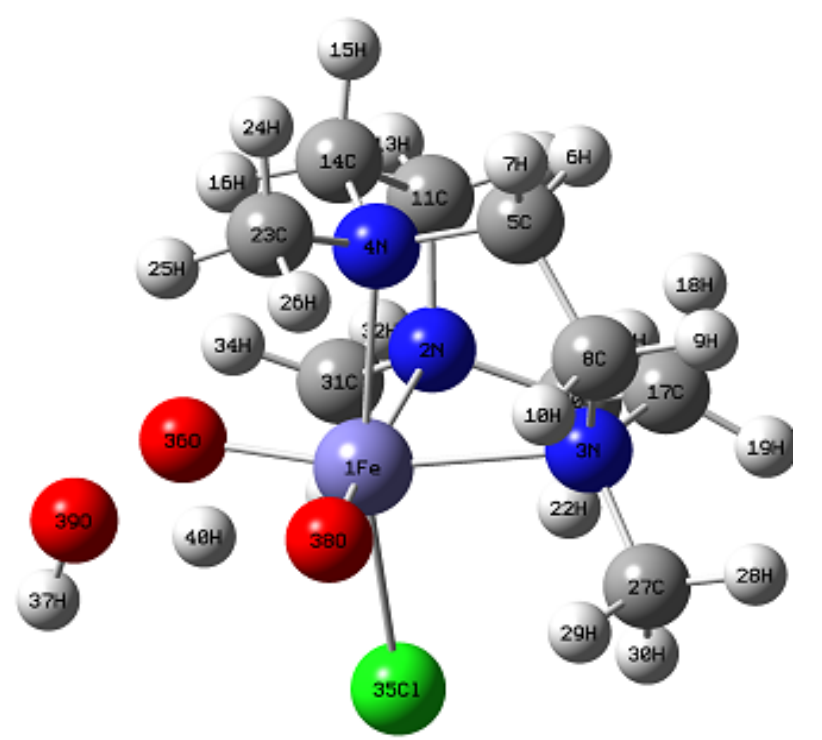

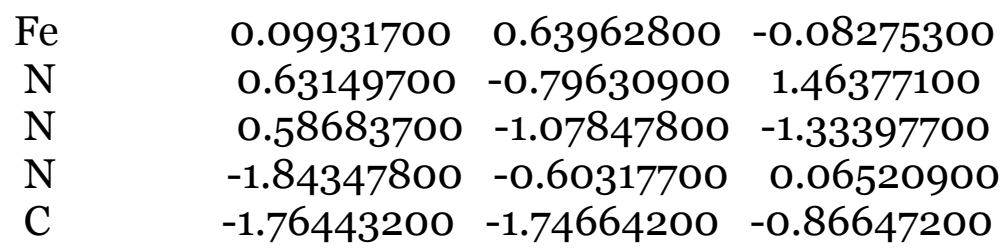




\begin{tabular}{|c|c|c|c|}
\hline $\mathrm{H}$ & -1.54857000 & -2.66214200 & -0.30621500 \\
\hline $\mathrm{H}$ & -2.73522400 & -1.91438900 & -1.35138800 \\
\hline $\mathrm{C}$ & -0.71354700 & -1.48870100 & -1.92629900 \\
\hline $\mathrm{H}$ & -0.57340500 & -2.38024000 & -2.55576500 \\
\hline $\mathrm{H}$ & -1.02451900 & -0.66000400 & -2.57264500 \\
\hline $\mathrm{C}$ & -0.54608300 & -1.62224300 & 1.87921100 \\
\hline $\mathrm{H}$ & -0.44952600 & -2.61651900 & 1.43191800 \\
\hline $\mathrm{H}$ & -0.51531200 & -1.77375500 & 2.96445100 \\
\hline $\mathrm{C}$ & -1.85682400 & -0.98055000 & 1.48518100 \\
\hline $\mathrm{H}$ & -2.69128200 & -1.66277500 & 1.71754800 \\
\hline $\mathrm{H}$ & -2.01055700 & -0.05412800 & 2.05087100 \\
\hline $\mathrm{C}$ & 1.20196100 & -2.16406800 & -0.50824900 \\
\hline $\mathrm{H}$ & 0.46680200 & -2.96314100 & -0.37552400 \\
\hline $\mathrm{H}$ & 2.04093500 & -2.61183600 & -1.05332600 \\
\hline $\mathrm{C}$ & 1.68156300 & -1.63485000 & 0.82640600 \\
\hline $\mathrm{H}$ & 1.95969600 & -2.46554100 & 1.49305700 \\
\hline $\mathrm{H}$ & 2.55812800 & -0.99358600 & 0.69253500 \\
\hline $\mathrm{C}$ & -2.97020700 & 0.27579300 & -0.25865200 \\
\hline $\mathrm{H}$ & -3.93098500 & -0.23041200 & -0.07537900 \\
\hline $\mathrm{H}$ & -2.90373300 & 1.18137900 & 0.35077500 \\
\hline $\mathrm{H}$ & -2.90877800 & 0.56493900 & -1.31154000 \\
\hline $\mathrm{C}$ & 1.49592600 & -0.67825200 & -2.42437900 \\
\hline $\mathrm{H}$ & 1.60657100 & -1.50079400 & -3.14523400 \\
\hline $\mathrm{H}$ & 1.07955900 & 0.20027400 & -2.92478100 \\
\hline $\mathrm{H}$ & 2.46984300 & -0.41784700 & -2.00572200 \\
\hline $\mathrm{C}$ & 1.17926500 & -0.09023800 & 2.63747100 \\
\hline $\mathrm{H}$ & 1.53743400 & -0.81403400 & 3.38284100 \\
\hline $\mathrm{H}$ & 2.00094300 & 0.55341600 & 2.31417900 \\
\hline $\mathrm{H}$ & 0.39158600 & 0.53001300 & 3.07454400 \\
\hline $\mathrm{Cl}$ & 2.48343500 & 1.47150200 & -0.11027200 \\
\hline $\mathrm{O}$ & -0.51260400 & 1.76945000 & 1.14605700 \\
\hline $\mathrm{H}$ & -0.73426000 & 3.87832800 & 0.08485300 \\
\hline $\mathrm{O}$ & -0.46962300 & 1.53103700 & -1.43338200 \\
\hline $\mathrm{O}$ & -1.48766500 & $3 \cdot 30431600$ & -0.13414500 \\
\hline $\mathrm{H}$ & -0.99659800 & 2.45277400 & -0.91243000 \\
\hline
\end{tabular}




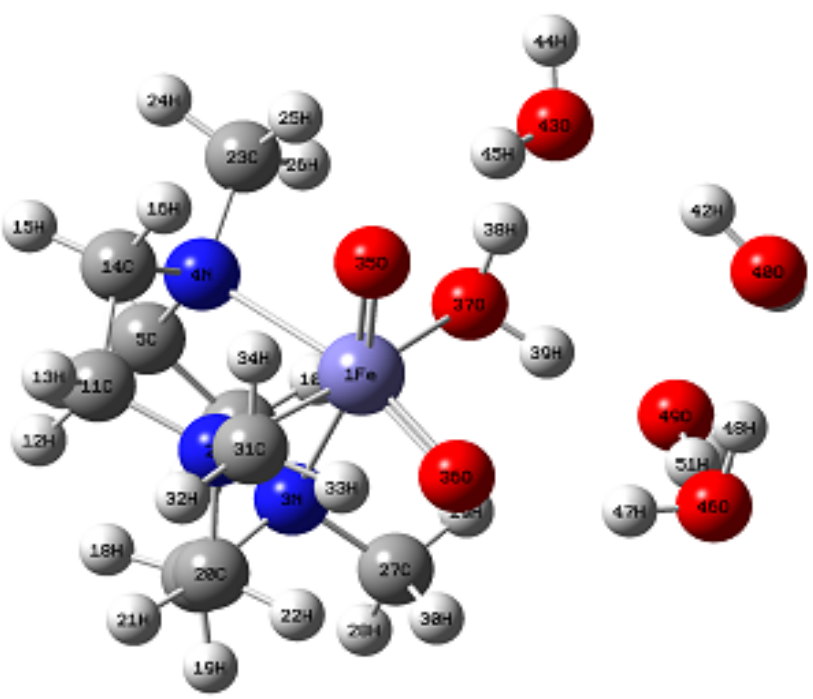

$\begin{array}{llll}\mathrm{Fe} & -0.02900000 & 0.10400000 & 0.30900000 \\ \mathrm{~N} & -1.66500000 & -0.45800000 & 1.46800000 \\ \mathrm{~N} & -1.00300000 & -1.34400000 & -1.13300000 \\ \mathrm{~N} & -1.62500000 & 1.36500000 & -0.71000000 \\ \mathrm{C} & -2.34000000 & 0.62000000 & -1.77700000 \\ \mathrm{H} & -3.30100000 & 0.27100000 & -1.38800000 \\ \mathrm{H} & -2.58400000 & 1.28700000 & -2.61200000 \\ \mathrm{C} & -1.49700000 & -0.53800000 & -2.26800000 \\ \mathrm{H} & -2.06600000 & -1.15600000 & -2.97800000 \\ \mathrm{H} & -0.61200000 & -0.16200000 & -2.79100000 \\ \mathrm{C} & -2.85500000 & 0.41400000 & 1.19600000 \\ \mathrm{H} & -3.58400000 & -0.17100000 & 0.63000000 \\ \mathrm{H} & -3.33600000 & 0.66800000 & 2.14500000 \\ \mathrm{C} & -2.47500000 & 1.67800000 & 0.45300000 \\ \mathrm{H} & -3.38200000 & 2.22500000 & 0.15600000 \\ \mathrm{H} & -1.88100000 & 2.32800000 & 1.10200000 \\ \mathrm{C} & -2.09500000 & -2.03100000 & -0.39400000 \\ \mathrm{H} & -3.05800000 & -1.62600000 & -0.71600000 \\ \mathrm{H} & -2.11800000 & -3.09600000 & -0.65200000 \\ \mathrm{C} & -1.91600000 & -1.88000000 & 1.10300000 \\ \mathrm{H} & -2.80100000 & -2.24800000 & 1.64000000 \\ \mathrm{H} & -1.04400000 & -2.44100000 & 1.44600000 \\ \mathrm{C} & -1.02500000 & 2.59500000 & -1.24500000 \\ \mathrm{H} & -1.79700000 & 3.32100000 & -1.53100000 \\ \mathrm{H} & -0.38000000 & 3.03100000 & -0.47800000 \\ \mathrm{H} & -0.42100000 & 2.35100000 & -2.12100000 \\ \mathrm{C} & -0.00900000 & -2.32600000 & -1.59100000 \\ \mathrm{H} & -0.45500000 & -3.03200000 & -2.30300000 \\ \mathrm{H} & 0.82400000 & -1.81000000 & -2.07200000 \\ \mathrm{H} & 0.38400000 & -2.86500000 & -0.72700000 \\ \mathrm{C} & -1.25500000 & -0.36300000 & 2.88700000 \\ \mathrm{H} & -2.04200000 & -0.77300000 & 3.52900000 \\ \mathrm{H} & -0.32700000 & -0.92000000 & 3.02200000 \\ \mathrm{H} & -1.07300000 & 0.68300000 & 3.13200000 \\ \mathrm{O} & 0.28200000 & 1.43200000 & 1.22800000 \\ \mathrm{O} & 0.86200000 & -1.14100000 & 0.88800000 \\ \mathrm{O} & 1.19500000 & 0.61200000 & -1.20600000 \\ & & & \\ & & & \end{array}$




$\begin{array}{llll}\mathrm{H} & 1.64600000 & 1.44600000 & -0.92800000 \\ \mathrm{H} & 1.94800000 & -0.05400000 & -1.32300000 \\ \mathrm{O} & 4.51100000 & 0.71800000 & 0.58000000 \\ \mathrm{H} & 4.55900000 & 0.46500000 & -0.35000000 \\ \mathrm{H} & 3.94500000 & 1.50200000 & 0.57600000 \\ \mathrm{O} & 2.41900000 & 2.63900000 & 0.11300000 \\ \mathrm{H} & 2.49700000 & 3.59400000 & 0.03400000 \\ \mathrm{H} & 1.78500000 & 2.46100000 & 0.83400000 \\ \mathrm{O} & 3.58600000 & -1.82600000 & 1.00700000 \\ \mathrm{H} & 2.68300000 & -1.73700000 & 1.34400000 \\ \mathrm{H} & 3.98700000 & -0.95200000 & 1.19200000 \\ \mathrm{O} & 3.24000000 & -0.96300000 & -1.44500000 \\ \mathrm{H} & 3.46600000 & -1.56400000 & -2.15900000 \\ \mathrm{H} & 3.37400000 & -1.45400000 & -0.57900000\end{array}$

TS for four explicit water molecules

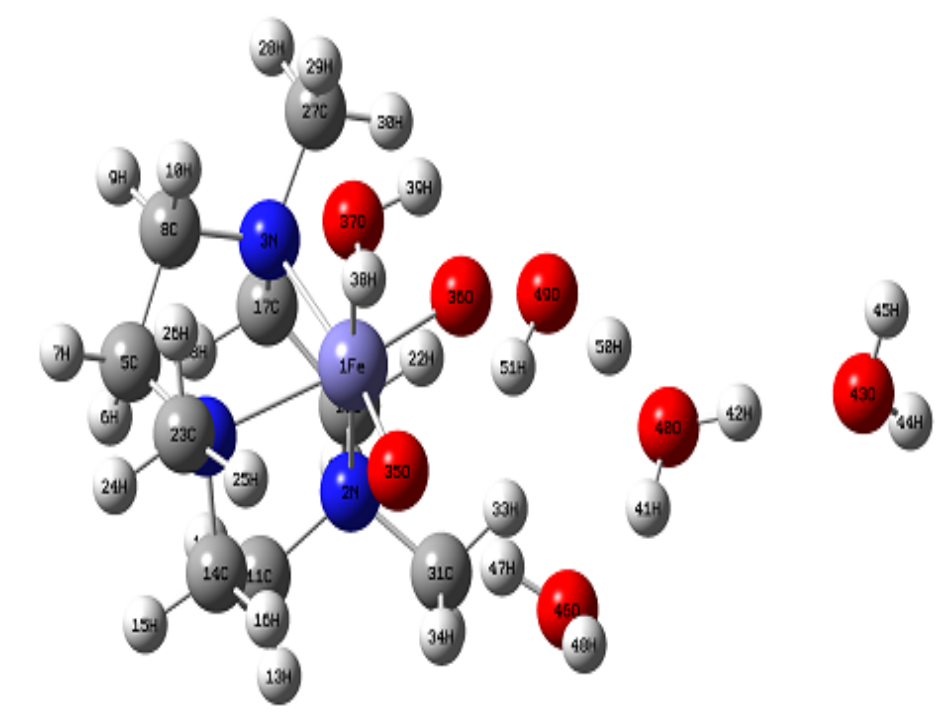

$\begin{array}{cccc}\mathrm{Fe} & -0.35900000 & -0.07900000 & -0.38900000 \\ \mathrm{~N} & -0.43400000 & 0.63200000 & 1.51000000 \\ \mathrm{~N} & -1.76600000 & -1.55900000 & 0.34900000 \\ \mathrm{~N} & -2.19500000 & 1.00800000 & -0.66300000 \\ \mathrm{C} & -3.37500000 & 0.18800000 & -0.28300000 \\ \mathrm{H} & -3.68900000 & 0.45800000 & 0.72900000 \\ \mathrm{H} & -4.22700000 & 0.41600000 & -0.93300000 \\ \mathrm{C} & -3.03000000 & -1.28400000 & -0.36700000 \\ \mathrm{H} & -3.85400000 & -1.90100000 & 0.02000000 \\ \mathrm{H} & -2.86500000 & -1.57300000 & -1.41100000 \\ \mathrm{C} & -1.54100000 & 1.63800000 & 1.62300000 \\ \mathrm{H} & -2.38100000 & 1.17400000 & 2.14700000 \\ \mathrm{H} & -1.21100000 & 2.47300000 & 2.24900000 \\ \mathrm{C} & -1.95900000 & 2.14500000 & 0.25800000 \\ \mathrm{H} & -2.85100000 & 2.78300000 & 0.34400000 \\ \mathrm{H} & -1.14900000 & 2.72700000 & -0.19000000 \\ \mathrm{C} & -1.88000000 & -1.35300000 & 1.82000000 \\ \mathrm{H} & -2.81400000 & -0.82700000 & 2.03700000 \\ \mathrm{H} & -1.95100000 & -2.31700000 & 2.33600000 \\ \mathrm{C} & -0.68500000 & -0.57900000 & 2.33900000 \\ \mathrm{H} & -0.82800000 & -0.29500000 & 3.39100000 \\ \mathrm{H} & 0.22400000 & -1.18400000 & 2.26500000\end{array}$




$\begin{array}{lccc}\mathrm{C} & -2.32000000 & 1.49100000 & -2.04700000 \\ \mathrm{H} & -3.15700000 & 2.19300000 & -2.14900000 \\ \mathrm{H} & -1.38300000 & 1.97900000 & -2.32200000 \\ \mathrm{H} & -2.49500000 & 0.64000000 & -2.71100000 \\ \mathrm{C} & -1.30400000 & -2.92800000 & 0.05800000 \\ \mathrm{H} & -2.03100000 & -3.66900000 & 0.41600000 \\ \mathrm{H} & -1.17900000 & -3.04500000 & -1.02000000 \\ \mathrm{H} & -0.33500000 & -3.08700000 & 0.53300000 \\ \mathrm{C} & 0.85800000 & 1.23300000 & 1.90100000 \\ \mathrm{H} & 0.84200000 & 1.49100000 & 2.96700000 \\ \mathrm{H} & 1.65200000 & 0.51200000 & 1.70200000 \\ \mathrm{H} & 1.03400000 & 2.12800000 & 1.30500000 \\ \mathrm{O} & 0.49600000 & 1.23300000 & -1.08000000 \\ \mathrm{O} & 0.94500000 & -1.12500000 & 0.00700000 \\ \mathrm{O} & -0.33100000 & -0.97900000 & -2.29500000 \\ \mathrm{H} & -0.04000000 & -0.19600000 & -2.79100000 \\ \mathrm{H} & 0.49200000 & -1.48900000 & -2.16300000 \\ \mathrm{O} & 3.87900000 & -0.18800000 & 0.46800000 \\ \mathrm{H} & 3.74600000 & 0.77700000 & 0.30200000 \\ \mathrm{H} & 4.82800000 & -0.39600000 & 0.30800000 \\ \mathrm{O} & 6.50900000 & -0.68400000 & 0.00200000 \\ \mathrm{H} & 7.08400000 & -0.72100000 & 0.77100000 \\ \mathrm{H} & 6.76100000 & -1.44100000 & -0.53300000 \\ \mathrm{O} & 2.88900000 & 2.19500000 & -0.26600000 \\ \mathrm{H} & 1.98400000 & 1.94100000 & -0.58100000 \\ \mathrm{H} & 3.25200000 & 2.77500000 & -0.93900000 \\ \mathrm{O} & 2.24500000 & -0.88900000 & -1.14000000 \\ \mathrm{H} & 3.06300000 & -0.67600000 & -0.38200000 \\ \mathrm{H} & 1.92300000 & 0.02300000 & -1.33100000\end{array}$

$\mathrm{TS} 2(4 \mathrm{wt})\left[\mathrm{Fe}\left(\mathrm{Me}_{3} \mathrm{tacn}\right)\left(\mathrm{H}_{2} \mathrm{O}\right)(\mathrm{OOH})(\mathrm{O})\right]^{+} \cdot \mathrm{H}_{3} \mathrm{O}^{+}{ }_{2} \mathbf{2 H}_{2} \mathrm{O}$

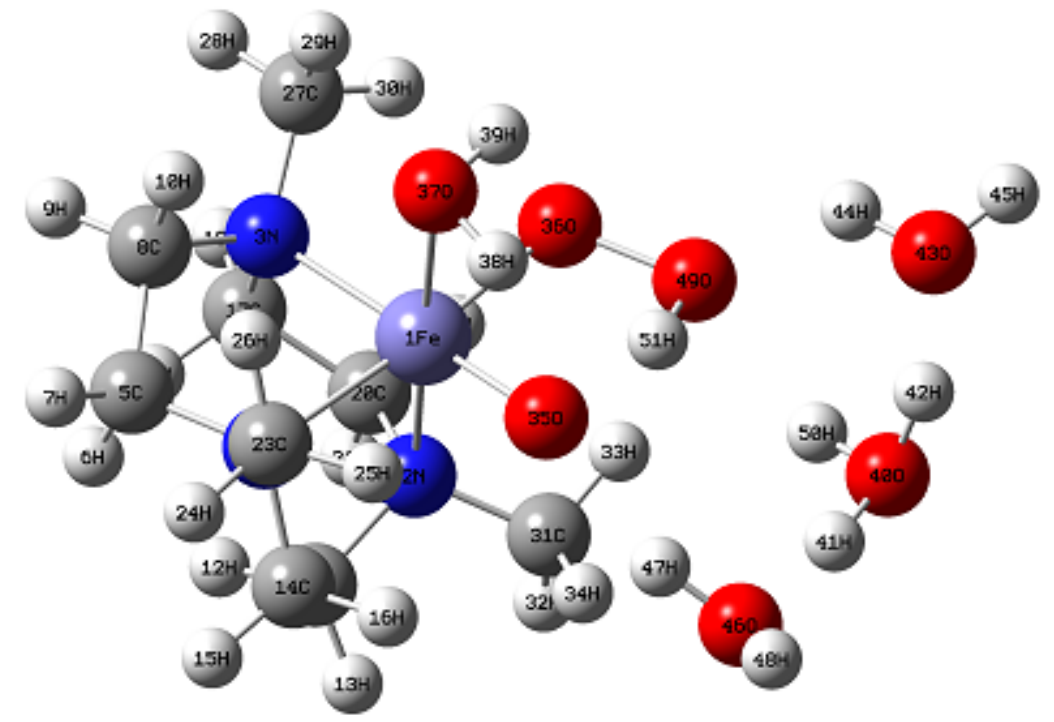

\begin{tabular}{|c|c|c|c|}
\hline $\mathrm{Fe}$ & -0.32100000 & -0.25400000 & -0.30200000 \\
\hline $\mathrm{N}$ & -0.42900000 & 0.72600000 & 1.47900000 \\
\hline $\mathrm{N}$ & -2.06400000 & -1.24800000 & 0.33900000 \\
\hline $\mathrm{N}$ & -1.65300000 & 1.18400000 & -0.98000000 \\
\hline $\mathrm{C}$ & -3.05300000 & 0.72500000 & -0.7520000 \\
\hline $\mathrm{H}$ & -3.44600000 & 1.20300000 & 0.1500000 \\
\hline
\end{tabular}




\begin{tabular}{|c|c|c|c|}
\hline $\mathrm{H}$ & -3.69900000 & 1.05300000 & -1.57300000 \\
\hline $\mathrm{C}$ & -3.08800000 & -0.78500000 & -0.62900000 \\
\hline $\mathrm{H}$ & -4.09300000 & -1.13300000 & -0.34800000 \\
\hline $\mathrm{H}$ & -2.83000000 & -1.24500000 & -1.58900000 \\
\hline $\mathrm{C}$ & -1.23600000 & 1.97300000 & 1.30600000 \\
\hline $\mathrm{H}$ & -2.24200000 & 1.80600000 & 1.70400000 \\
\hline $\mathrm{H}$ & -0.80600000 & 2.78500000 & 1.90200000 \\
\hline $\mathrm{C}$ & -1.27700000 & 2.35700000 & -0.15600000 \\
\hline $\mathrm{H}$ & -1.95800000 & 3.20400000 & -0.32300000 \\
\hline $\mathrm{H}$ & -0.27300000 & 2.63800000 & -0.49400000 \\
\hline $\mathrm{C}$ & -2.34600000 & -0.81100000 & 1.73600000 \\
\hline $\mathrm{H}$ & -3.14000000 & -0.05900000 & 1.72500000 \\
\hline $\mathrm{H}$ & -2.73300000 & -1.64900000 & 2.32700000 \\
\hline $\mathrm{C}$ & -1.08500000 & -0.26900000 & 2.37300000 \\
\hline $\mathrm{H}$ & -1.29600000 & 0.16900000 & 3.35800000 \\
\hline $\mathrm{H}$ & -0.35600000 & -1.07700000 & 2.49800000 \\
\hline $\mathrm{C}$ & -1.43500000 & 1.47800000 & -2.41000000 \\
\hline $\mathrm{H}$ & -2.05100000 & 2.32800000 & -2.73100000 \\
\hline $\mathrm{H}$ & -0.37600000 & 1.69200000 & -2.55500000 \\
\hline $\mathrm{H}$ & -1.70100000 & 0.59900000 & -3.00100000 \\
\hline $\mathrm{C}$ & -1.91500000 & -2.71200000 & 0.27500000 \\
\hline $\mathrm{H}$ & -2.83700000 & -3.21900000 & 0.59000000 \\
\hline $\mathrm{H}$ & -1.68200000 & -3.00300000 & -0.75000000 \\
\hline $\mathrm{H}$ & -1.08600000 & -3.01700000 & 0.91700000 \\
\hline $\mathrm{C}$ & 0.90500000 & 1.04800000 & 2.01100000 \\
\hline $\mathrm{H}$ & 0.83000000 & 1.48800000 & 3.01400000 \\
\hline $\mathrm{H}$ & 1.48100000 & 0.12100000 & 2.05500000 \\
\hline $\mathrm{H}$ & 1.39600000 & 1.75200000 & 1.33700000 \\
\hline $\mathrm{O}$ & 1.07700000 & 0.59000000 & -1.04200000 \\
\hline $\mathrm{O}$ & 0.79200000 & -1.67700000 & 0.33900000 \\
\hline $\mathrm{O}$ & -0.19100000 & -1.31600000 & -2.08700000 \\
\hline $\mathrm{H}$ & 0.52800000 & -0.64500000 & -2.25200000 \\
\hline $\mathrm{H}$ & 0.30000000 & -2.05300000 & -1.68000000 \\
\hline $\mathrm{O}$ & 4.28400000 & 0.35000000 & 0.81900000 \\
\hline $\mathrm{H}$ & 3.91000000 & 1.19800000 & 0.30700000 \\
\hline $\mathrm{H}$ & 4.60300000 & -0.36400000 & 0.14000000 \\
\hline $\mathrm{O}$ & 4.62400000 & -1.55100000 & -0.81300000 \\
\hline $\mathrm{H}$ & 3.73500000 & -1.91900000 & -0.62800000 \\
\hline $\mathrm{H}$ & 5.26000000 & -2.25800000 & -0.66300000 \\
\hline $\mathrm{O}$ & 3.17400000 & 2.28400000 & -0.30800000 \\
\hline $\mathrm{H}$ & 2.35100000 & 1.87800000 & -0.68300000 \\
\hline $\mathrm{H}$ & 3.60800000 & 2.76000000 & -1.02300000 \\
\hline $\mathrm{O}$ & 2.19800000 & -1.25000000 & 0.21300000 \\
\hline $\mathrm{H}$ & 3.49100000 & -0.08600000 & 1.20000000 \\
\hline $\mathrm{H}$ & 1.99300000 & -0.46100000 & -0.42900000 \\
\hline
\end{tabular}

$\left[\mathrm{Fe}\left(\mathrm{Me}_{3} \operatorname{tacn}\right)\left(\mathrm{H}_{2} \mathrm{O}\right)(\mathrm{OOH})(\mathrm{OH})\right]^{+}{ }^{+3} \mathrm{H}_{2} \mathrm{O}$ 


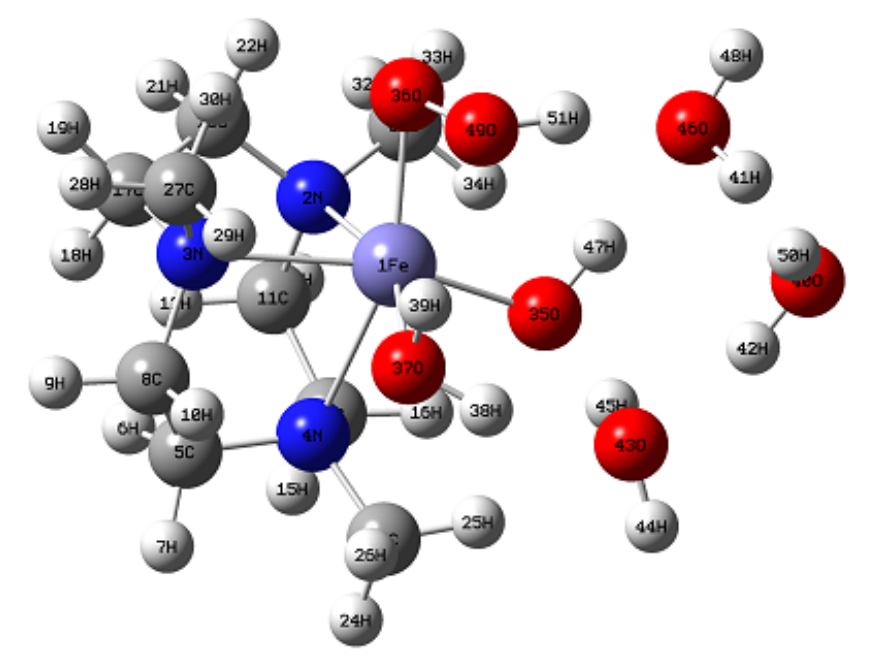

$\begin{array}{lccc}\mathrm{Fe} & -0.11191723 & -0.14103399 & 0.11673147 \\ \mathrm{~N} & 1.43344612 & -1.21697924 & -1.15731120 \\ \mathrm{~N} & 1.75858816 & -0.07234860 & 1.45821469 \\ \mathrm{~N} & 1.14447271 & 1.58671108 & -0.74912718 \\ \mathrm{C} & 2.32877917 & 1.90064286 & 0.09190042 \\ \mathrm{H} & 3.22588599 & 1.50207209 & -0.38776231 \\ \mathrm{H} & 2.47880094 & 2.98450509 & 0.14221146 \\ \mathrm{C} & 2.17361310 & 1.34514518 & 1.49289586 \\ \mathrm{H} & 3.11251434 & 1.47083050 & 2.05214560 \\ \mathrm{H} & 1.39203755 & 1.88875741 & 2.03126800 \\ \mathrm{C} & 2.21093596 & -0.26393501 & -1.99029338 \\ \mathrm{H} & 3.21394246 & -0.15489753 & -1.57007886 \\ \mathrm{H} & 2.35414062 & -0.67439347 & -2.99575289 \\ \mathrm{C} & 1.51221338 & 1.07530603 & -2.08666575 \\ \mathrm{H} & 2.14748306 & 1.79581390 & -2.62281738 \\ \mathrm{H} & 0.57529855 & 0.97122310 & -2.64290476 \\ \mathrm{C} & 2.81316457 & -0.95183274 & 0.88087580 \\ \mathrm{H} & 3.59481545 & -0.32431827 & 0.44519659 \\ \mathrm{H} & 3.30320315 & -1.52150471 & 1.67807490 \\ \mathrm{C} & 2.26698440 & -1.91528108 & -0.15645634 \\ \mathrm{H} & 3.09996959 & -2.44706138 & -0.63988813 \\ \mathrm{H} & 1.62977876 & -2.66714639 & 0.31852841 \\ \mathrm{C} & 0.29529960 & 2.77737686 & -0.89289870 \\ \mathrm{H} & 0.81919538 & 3.57138425 & -1.44195254 \\ \mathrm{H} & -0.61019302 & 2.50723620 & -1.43792980 \\ \mathrm{H} & 0.02657959 & 3.15765095 & 0.09464476 \\ \mathrm{C} & 1.39323835 & -0.53376779 & 2.80491393 \\ \mathrm{H} & 2.25112701 & -0.48761136 & 3.48937630 \\ \mathrm{H} & 0.59904087 & 0.09899625 & 3.20731468 \\ \mathrm{H} & 1.02814805 & -1.55997252 & 2.74363987 \\ \mathrm{C} & 0.71950981 & -2.17811781 & -2.01157910 \\ \mathrm{H} & 1.42414378 & -2.79453542 & -2.58566689 \\ \mathrm{H} & 0.09419651 & -2.82120683 & -1.38986496 \\ \mathrm{H} & 0.07660627 & -1.62802900 & -2.70204325 \\ \mathrm{O} & -1.38149817 & 0.19013090 & -1.27090788 \\ \mathrm{O} & -0.45544044 & -1.93691823 & 0.76829439 \\ \mathrm{O} & -0.93214903 & 1.33723935 & 1.52592935 \\ \mathrm{H} & -1.68974904 & 1.75375986 & 1.04133377 \\ & & & \\ & & & \end{array}$




$\begin{array}{llll}\mathrm{H} & -1.37176523 & 0.76413513 & 2.17033716 \\ \mathrm{O} & -5.11552592 & 0.20860194 & 0.08310620 \\ \mathrm{H} & -4.10720401 & -1.10031102 & -0.39029864 \\ \mathrm{H} & -4.47815402 & 0.95147526 & 0.06227586 \\ \mathrm{O} & -2.97430480 & 1.96460455 & -0.12269858 \\ \mathrm{H} & -3.08049643 & 2.81153233 & -0.56810126 \\ \mathrm{H} & -2.46210419 & 1.37651851 & -0.74348251 \\ \mathrm{O} & -3.36651854 & -1.72545499 & -0.59638317 \\ \mathrm{H} & -1.97474360 & -0.57653098 & -1.36244889 \\ \mathrm{H} & -3.77227165 & -2.57666202 & -0.78415214 \\ \mathrm{O} & -1.64295237 & -1.37682761 & 1.40492021 \\ \mathrm{H} & -5.32942387 & 0.10482353 & 1.01527482 \\ \mathrm{H} & -2.35181987 & -1.61555875 & 0.74182998\end{array}$

